\title{
Finite-size scaling properties of random transverse-field Ising chains : Comparison between canonical and microcanonical ensembles for the disorder
}

\author{
Cécile Monthus \\ Service de Physique Théorique, Unité de recherche associée au CNRS, \\ DSM/CEA Saclay, 91191 Gif-sur-Yvette, France
}

\begin{abstract}
The Random Transverse Field Ising Chain is the simplest disordered model presenting a quantum phase transition at $T=0$. We compare analytically its finite-size scaling properties in two different ensembles for the disorder (i) the canonical ensemble, where the disorder variables are independent (ii) the microcanonical ensemble, where there exists a global constraint on the disorder variables. The observables under study are the surface magnetization, the correlation of the two surface magnetizations, the gap and the end-to-end spin-spin correlation $C(L)$ for a chain of length $L$. At criticality, each observable decays typically as $e^{-w \sqrt{L}}$ in both ensembles, but the probability distributions of the rescaled variable $w$ are different in the two ensembles, in particular in their asymptotic behaviors. As a consequence, the dependence in $L$ of averaged observables differ in the two ensembles. For instance, the correlation $C(L)$ decays algebraically as $1 / L$ in the canonical ensemble, but sub-exponentially as $e^{-c L^{1 / 3}}$ in the microcanonical ensemble. Off criticality, probability distributions of rescaled variables are governed by the critical exponent $\nu=2$ in both ensembles, but the following observables are governed by the exponent $\tilde{\nu}=1$ in the microcanonical ensemble, instead of the exponent $\nu=2$ in the canonical ensemble (a) in the disordered phase : the averaged surface magnetization, the averaged correlation of the two surface magnetizations and the averaged end-to-end spin-spin correlation (b) in the ordered phase : the averaged gap. In conclusion, the measure of the rare events that dominate various averaged observables can be very sensitive to the microcanonical constraint.
\end{abstract}

\section{INTRODUCTION}

\section{A. Microcanonical ensemble versus canonical ensemble for a finite disordered sample}

In the study of disordered systems, it is usual to consider that the random variables defining the disorder in a given sample are independent : following 1, 2], this procedure will be called here the "canonical ensemble" (this procedure is also called the "grand-canonical ensemble" in [3, 4, 5, 6] ). However, it has been argued in [3] that it is much more interesting in some cases to consider the so called "microcanonical ensemble" as in 1, 2] ( this procedure is called the "canonical ensemble" in [3, 4, 5, [6]) : in the microcanonical ensemble, there exists a global constraint on the random variables defining the disorder in a given sample of $N$ sites. The first important example concerns a pure system with a fraction $p \in[0,1]$ of impurities [3] , i.e. the disorder is characterized by a binary distribution : in the canonical ensemble obtained by putting independently on each site an impurity with probability $p$, the total number of impurities presents fluctuations of order $\sqrt{N}$ around its mean value $p N$, whereas in the microcanonical ensemble, the total number of impurities is fixed to be exactly $p N$ and presents no fluctuations at all, i.e. the remaining disorder only concerns the positions of the impurities. It is thus clear that the microcanonical ensemble is much less disordered that the canonical ensemble, and indeed, it may yield a spectacular noise reduction in numerical studies [3, 4]. Another example where the ensemble dependence has been under study recently is the Random Transverse Field Ising Chain [1, 2], defined by the Hamiltonian

$$
H=-\sum_{i} J_{i} \sigma_{i}^{z} \sigma_{i+1}^{z}-\sum_{i} h_{i} \sigma_{i}^{x}
$$

where the couplings $J_{i}>0$ and the fields $h_{i}>0$ are random variables (the signs can be fixed via a gauge transformation). This model is of great interest because it is the simplest disordered model presenting a quantum phase transition at $T=0:$ the critical point situated at

$$
[\ln h]_{a v}=[\ln J]_{a v}
$$

separates a ferromagnetic phase $\left([\ln h]_{a v}<[\ln J]_{a v}\right)$ from a disordered phase $\left([\ln h]_{a v}>[\ln J]_{a v}\right)$. This model can be studied in great details via a disorder-dependent real space RG analysis 7], which agrees with previous exact results [8, [9] whenever they can be compared. In numerical studies of the quantum critical point on systems of linear size $L$, the usual canonical ensemble has been compared to the microcanonical ensemble defined by the global constraint 


$$
\sum_{i=1}^{L}\left(\ln J_{i}-\ln h_{i}\right)=0
$$

(with one fixed boundary condition so that there are exactly the same number $L$ of random bonds and random fields). In the canonical ensemble, the quantity on the left in equation (3) presents fluctuations of order $\sqrt{L}$ around its mean value zero. The samples of the microcanonical ensemble are thus 'closer to criticality' in some sense than the samples of the canonical ensemble. So in both examples, the microcanonical constraint appears as an opportunity to obtain numerical results with much less fluctuations.

Now the question is : is that interesting from the physics point of view? On one hand, it has been strongly argued in 3, 4] that the microcanonical ensemble should be preferred to the canonical ensemble, because the latter introduces an "extra noise" that may hid the "intrinsic" properties of the system. On the other hand, it seems that the fluctuations of order $\sqrt{l}$ for the sum of $l$ random variables are precisely an essential property of disordered systems, and indeed these fluctuations appear in the Harris argument 10] on the relevance of disorder near critical points, in the Imry-Ma argument 11] for random field Ising models, and in the Chayes et al. theorem [12] for the bound $\nu \geq 2 / d$. Moreover, if one divides the system of size $L$ into two halfs of size $L / 2$, each half will present fluctuations of order $\sqrt{L}$ in both ensembles : in the canonical ensemble, these two halfs are independent, whereas in the microcanonical ensemble, the two halfs are completely correlated, i.e. they have exactly opposite fluctuations. From this point of view, the microcanonical constraint can thus appear to be quite artificial or even biased.

Of course, it seems a priori natural to expect that these two ensembles should be equivalent in the thermodynamic limit, as was shown in [6] for the case of a random classical ferromagnet. However, it is clear on the two examples above that their finite-size properties can be very different. But since the finite-size scaling theory of phase transitions relates the thermodynamic exponents to finite-size effects obtained in numerical simulations, the discussion about these two ensembles actually leads to the general problem of the finite-size scaling theory for disordered systems [3, 4, 5].

\section{B. Relation with the finite-size scaling theory for disordered systems}

To generalize the ideas of the finite-size scaling theory developed for pure systems to the case of disordered systems, the central question is [3, 4, 5] : when the numerical simulations provide values for some observable, like the susceptibility $\chi(i, L)$, for various samples $(i)$ and various sizes $L$, what is the best procedure to analyze these data? The usual procedure consists in averaging the observable $\chi_{m}(L)=\left\langle\chi(i, L)>_{i}\right.$ over the samples $(i)$ at fixed size $L$ and in analyzing the dependence in $L$ of the mean values $\chi_{m}(L)$ as in the finite size scaling theory of pure systems. However, as explained in details in 3, 4, 5] with illuminating numerical examples, the correct procedure to analyze the data consists in a finite-size scaling analysis for each given sample. In particular, one has to defined a pseudo-critical temperature $T_{c}(i, L)$ for each sample, either by the maximum of the susceptibility [4, 5] or by a distance minimizing procedure [4]. Indeed, these studies have shown that the fluctuations of $\chi(i, L)$ over the samples are mainly due to fluctuations in $T_{c}(i, L)$, and that the use of the variable $\left(T-T_{c}(i, L)\right)$ in the finite-size scaling analysis yields much more accurate results (see for instance Fig.2 and Fig.3 in [4]). Within this point of view, the advantage of the microcanonical ensemble with respect to the canonical ensemble is justified if the microcanonical constraint determines $T_{c}(i, L)$ or at least reduces drastically its fluctuations over the samples, as it is the case for binary distributions $[\underline{3}, 4]$. For quantum phase transitions, the pseudo-critical temperature $T_{c}(i, L)$ of classical systems has to be replaced by the pseudo critical point [4]. In conclusion, the best justification for the microcanonical ensemble seems to be in systems where the microcanonical constraint actually corresponds to a constraint on the pseudo-critical temperature like in critical phenomena with a binary distribution [3, 4] or to a constraint on the pseudo critical point for quantum phase transitions as in the Random Transverse Field Ising model.

\section{Ensemble dependence in the RTFIC}

The ensemble dependence in the RTFIC has already been studied in [1, 2]. The first considered observable has been the surface magnetization $m_{S}$ : in particular, its averaged value over the samples was found to present different scaling in $L$ at criticality in the two ensembles [1, 2] : it decays as $1 / \sqrt{L}$ in the canonical ensemble and as $1 / L$ in the microcanonical sample [1, 2]. However, the interpretation that should be given to this difference is still under debate [1, 2] : in [1] it was interpreted as the presence of the two different correlation exponents $\nu=2$ and $\tilde{\nu}=1$ [7], whereas 
in [2] it was interpreted as the same exponent $\nu=2$ in both ensembles, with an accidental vanishing of an amplitude in the microcanonical ensemble.

In [2], other observables have been numerically compared at criticality in the two ensembles, from the point of view of probability distributions as well as averaged values. The considered observables are the middle-point magnetization, the end-to-end spin-spin correlation

$$
C(L) \equiv<\sigma_{1}^{z} \sigma_{L}^{z}>
$$

the gap, i.e. the energy difference between the two lowest levels

$$
\Delta(L) \equiv E_{1}-E_{0}
$$

and also the correlation $\left(m_{1}^{s} m_{L}^{s}\right)$ between the two surface magnetizations [2] where each surface magnetization corresponds to the magnetization at one end when the spin at the other end is fixed

$$
\begin{aligned}
& m_{1}^{s} \equiv<\sigma_{1}^{z}>\left.\right|_{\sigma_{L}^{z}=1} \\
& m_{L}^{s} \equiv<\sigma_{L}^{z}>\left.\right|_{\sigma_{1}^{z}=1}
\end{aligned}
$$

We refer to [2] for a detailed discussion on the numerical results for each observable.

The aim of this paper is to compare analytically the behavior of these various observables in both ensembles, at criticality as well as off-criticality, with a particular attention to the averaged values, that are governed by rare events.

The paper is organized as follows. In Section【 we recall the relation between the surface magnetization and Kesten random variables, and introduce the important notations. In Section III we show that the limit distributions for the surface magnetization obtained in [2] can be understood as resulting from a saddle point method in each sample. In Section [V] and $\nabla$ we compute the exact distribution and the exact averaged value of the surface magnetization, in the canonical and microcanonical ensembles respectively, via path-integral methods. In Section VI we apply the saddle-point method to compute the limit distributions for the correlation of the two surface magnetizations in the two ensembles. In Section VII we compute via the real-space renormalization approach the probability distribution of the gap and of the spin-spin end-to-end correlation in the microcanonical ensemble and compare with the previously known results for the canonical ensemble [13]. Finally, the Section VIII] contains the conclusions and the appendixes some technical details.

\section{SURFACE MAGNETIZATION AND KESTEN RANDOM VARIABLES}

In this Section, we recall that the surface magnetization is a Kesten random variable, and introduce the important notations.

\section{A. Surface magnetization in each given sample}

Remarkably, the surface magnetization (6) in any given sample of size $(L+1)$ has the following exact expression [1, 2]

$$
m_{1}^{S}=\left[1+Z_{L}\right]^{-1 / 2}
$$

where

$$
Z_{L} \equiv \sum_{i=1}^{L} \prod_{j=1}^{i}\left(\frac{h_{i}}{J_{i}}\right)^{2}
$$

has a specific structure of a sum of products of random variables : we will call $Z_{L}$ a Kesten random variable 14 . This type of random variables also appears in the context of random walks in random media for various observables 15, 16, 17, 18], as well as in the random field Ising chain via the formulation with $2 \times 2$ random transfer matrices $19,20]$. 


\section{B. Exponent $\mu$ for discrete Kesten random variables}

It is actually convenient to rewrite the Kesten random variable (8) with a varying left boundary

$$
Z(a, b)=\sum_{i=a}^{b} \prod_{j=1}^{i} y_{j}
$$

where $y_{j}=\left(h_{i} / J_{i}\right)^{2}$ are the independent random variables. The fundamental property of $Z(a, b)$ is the recurrence equation

$$
Z(a, b)=y_{a}[1+Z(a+1, b)]
$$

where the random coefficient $y_{a}$ appears multiplicatively : $Z(a, b)$ is thus a multiplicative stochastic process.

One of the main outcome of the studies on the random variable $Z(a, b)$ is that, in the limit of infinite length $L=b-a \rightarrow \infty$, there exists a limit distribution $P_{\infty}(Z)$ if

$$
[\ln y]_{a v}<0
$$

Moreover, the limit distribution then presents the algebraic tail [14, 19, 20, 21]

$$
P_{\infty}(Z) \underset{Z \rightarrow \infty}{\sim} \frac{1}{Z^{1+\mu}}
$$

where the exponent $\mu$ is defined as the positive root $\mu>0$ of the equation

$$
\left[y^{\mu}\right]_{a v}=1
$$

In the field of random walks in random media, this exponent $\mu$ is known to govern the anomalous diffusion behavior $x \sim t^{\mu}$ in the domain $0<\mu<1$ [14, 17, 22, 23]. In the context of the RTFIC, the exponent $\mu$ defined by (13) has for analog the RG-invariant exponent $-(2 \Delta)[24]$ defined by $\left[(J / h)^{2 \Delta}\right]_{a v}=1$, and $z=1 /(2 \Delta)$ can be interpreted as a dynamical exponent. In the vicinity of the critical point $\Delta=0$, one may perform a series expansion in $\Delta$, to obtain the solution as $\Delta=\delta+O\left(\delta^{2}\right)$, where

$$
\delta=-\frac{\left[\ln \frac{J}{h}\right]_{a v}}{\left[\left(\ln \frac{J}{h}\right)^{2}\right]_{a v}-\left(\left[\ln \frac{J}{h}\right]_{a v}\right)^{2}}=\frac{[\ln h]_{a v}-[\ln J]_{a v}}{\operatorname{var}(\ln h)+\operatorname{var}(\ln J)}
$$

is the parameter introduced in [7] to measure the deviation from criticality in the real-space renormalization approach. The expression (14), which comes from an expansion in the two first cumulants of the variable $\ln (J / h)$, is thus exact if the variable $\ln (J / h)$ is Gaussian, and it is only an approximation near the critical point for all other distributions which present higher order cumulants.

\section{Continuous version of Kesten random variables}

The continuous version of the Kesten random variable (9]) is the exponential functional 22, 25]

$$
Z[a, b]=\int_{a}^{b} d x e^{-\int_{a}^{x} d y F(y)}
$$

where $\{F(x)\}$ is the random process corresponding to the random variables $\left(-\ln y_{i}\right)=-2 \ln \left(h_{i} / J_{i}\right)$ in the continuous limit. The analog of the recurrence equation (10) is the stochastic differential equation

$$
\partial_{a} Z[a, b]=F(a) Z[a, b]-1
$$

where the random process $F(x)$ appears multiplicatively, in contrast with usual Langevin equations where the noise appears additively. In the limit of infinite length $L=b-a \rightarrow \infty$, the condition (11) to have a limit distribution $P_{\infty}(Z)$ becomes a condition on the mean value of the process $F(x)$ that should be strictly positive

$$
F_{0} \equiv[F(x)]_{a v}>0
$$


The exponent $\mu$ (12) is now determined as the root of the equation (13)

$$
\left[e^{-\mu \int_{0}^{x} d y F(y)}\right]_{a v}=1
$$

for arbitrary $x$ as long as the process $F(x)$ has no correlation.

It is interesting to note that the exponential functional (15) actually determines the stationary flux $J_{L}[25,26,27$ that exists in a given Sinai sample $[0, L]$ between two fixed concentration $c_{0}$ and $c_{N}=0$ (i.e. particles are injected via a reservoir at $x=0$ and are removed when they arrive at the other boundary $x=N)$ : it is simply given by the inverse of the variable $Z_{L} \equiv Z[0, L]$

$$
J_{L}=\frac{c_{0}}{Z_{L}}
$$

In some sense, it is the simplest physical observable in the Sinai diffusion, as the surface magnetization is the simplest order parameter in the RTFIC : both can be expressed in a simple way in terms of the Kesten random variable of the sample.

\section{Case of a Brownian process}

The simplest process for $F(x)$ is of course the case where $F(x)$ is a biased Brownian motion

$$
\begin{aligned}
<F(x)> & =F_{0} \\
<F(x) F\left(x^{\prime}\right)>-F_{0}^{2} & =2 \sigma \delta\left(x-x^{\prime}\right)
\end{aligned}
$$

In this case, the exponent $\mu$ solution of [18) reads [22, 25]

$$
\mu=\frac{F_{0}}{\sigma}
$$

Here $\mu$ exactly coincides with $(-2 \delta)$ even away from criticality, as a consequence of the Gaussian distribution. The Brownian process actually corresponds to the fixed point of the real-space renormalization approach 7] and can thus be used to study the universal properties near the critical point.

\section{LIMIT DISTRIBUTIONS FOR THE SURFACE MAGNETIZATION IN THE LARGE $L$ LIMIT}

In this Section, we discuss the universal limit distributions of the random variable $\left(\ln m_{S}(L)\right)$ in the large $L$ limit near the critical point, which have been obtained in [2] via an approximation of the recurrence relation (10) for $\ln Z$ by an effective biased random walk with a reflecting boundary at the origin. Here we show that these analytic results [2] can actually be understood as resulting from a saddle point method in each sample. This point of view gives a clearer control on the validity of the approximation, and yields a better understanding of the limitations of the results obtained for the averaged surface magnetization that is governed by rare events. Moreover, this approach can be then generalized to obtain results for more complex quantities such as the correlation of the two surface magnetization as discussed in Section V1

\section{A. Saddle point method in each sample}

In the case of a Brownian process (21), the continuous version of the Kesten process (15) can be written as

$$
Z_{L}=\int_{0}^{L} d x e^{-U(x)}
$$

where the potential $U(x)=\int_{0}^{x} d y F(y)$ is a random walk of bias $F_{0}$. At the critical point $F_{0}=0$, this potential thus presents fluctuations of order $\sqrt{L}$ on the interval $[0, L]$. As a consequence, it seems natural to evaluate this integral in the large $L$ limit by the saddle-point method

$$
Z_{L} \underset{L \rightarrow \infty}{\simeq} e^{E_{L}} Z_{\text {valley }}
$$


where $\left(-E_{L}\right)<0$ is defined as the minimum reached by the process $U(x)$ on the interval $[0, L]$, and where $Z_{\text {valley }}$ represents the partition function of an infinitely deep Brownian valley, whose probability distribution was studied in [28]. At the critical point, the scaling $E_{L} \sim \sqrt{L}$ shows that there will exists a limit distribution for $\left(\ln Z_{L}\right) / \sqrt{L} \sim$ $\ln m_{L}^{s} / \sqrt{L}$, and that this limiting distribution is given by the limit distribution of $E_{L} / \sqrt{L}$. In the following, we study the distribution of the minimum $E_{L}$ at criticality and off criticality.

To study the probability distribution in the two ensembles, we thus need the following standard result : the probability for the biased random walk to go from $U_{0}$ to $U$ during $L$ in the presence of an absorbing boundary at $U=U_{a}$ reads from the methods of images

$$
G_{\left[U_{a},+\infty[\right.}^{\left(F_{0}\right)}\left(U, L \mid U_{0}\right)=\frac{1}{\sqrt{4 \pi \sigma L}}\left(e^{-\frac{\left(U-U_{0}-F_{0} l\right)^{2}}{4 \sigma L}}-e^{-\frac{F_{0}}{\sigma}\left(U_{0}-U_{a}\right)} e^{-\frac{\left(U+U_{0}-2 U_{a}-F_{0} L\right)^{2}}{4 \sigma L}}\right)
$$

The joint probability of the end-point $U$ and of the minimum value $U_{\min }=-E<0$ when starting at $U_{0}=0$ then reads

$$
\begin{aligned}
P_{L}^{\left(F_{0}\right)}(U, E) & =-\left[\partial_{U_{a}} G_{\left[U_{a},+\infty[\right.}^{\left(F_{0}\right)}(U, L \mid 0)\right]_{U_{a}=-E} \\
& =\theta(E>0) \theta(U>-E) \frac{(U+2 E)}{2 \sqrt{\pi}(\sigma L)^{3 / 2}} e^{-\frac{F_{0}}{\sigma} E} e^{-\frac{\left(U+2 E-F_{0} L\right)^{2}}{4 \sigma L}}
\end{aligned}
$$

\section{B. Distribution of $E_{L}$ in the canonical ensemble}

In the canonical ensemble, we need the partial law of $E$ when integrating over the end-point $U$ in (26)

$$
\begin{aligned}
{\left[P_{L}^{\left(F_{0}\right)}(E)\right]_{\text {cano }} } & =\int d U P_{L}^{\left(F_{0}\right)}(U, E) \\
& =\theta(E>0) e^{-\frac{F_{0}}{\sigma} E} \int_{E}^{+\infty} d V \frac{V}{2 \sqrt{\pi}(\sigma L)^{3 / 2}} e^{-\frac{\left(V-F_{0} L\right)^{2}}{4 \sigma L}}
\end{aligned}
$$

The final result reads after an integration by part and with the notation $F_{0}=\sigma \mu(22)$

$$
\mathcal{P}_{L}^{\text {cano }}(E)=\theta(E>0)\left[\frac{1}{\sqrt{\pi \sigma L}} e^{-\frac{(E+\mu \sigma L)^{2}}{4 \sigma L}}+\mu e^{-\mu E} \int_{\frac{(E+\mu \sigma L)}{\sqrt{4 \sigma L}}}^{+\infty} \frac{d z}{\sqrt{\pi}} e^{-z^{2}}\right]
$$

which exactly coincides with the formula (10) in [2] with the correspondence $\mu=-2 \bar{\delta}$ and $l=\sigma L$. Let us first consider the limit $L \rightarrow \infty$ with $\mu$ fixed :

For $\mu>0$, there exists a limit distribution for $E$ which is simply exponential

$$
\mathcal{P}_{\text {cano }}^{(\mu>0)}(E) \underset{L \stackrel{\sim}{\simeq} \theta}{\simeq} \theta(E>0) \mu e^{-\mu E}
$$

This distribution actually corresponds to the distribution of barriers against the bias [23, 29] in a Sinai potential.

For $\mu \leq 0$, the appropriate rescaled variable reads

$$
\mathcal{E} \equiv \frac{(E-|\mu| \sigma L)}{\sqrt{4 \sigma L}}
$$

For $\mu<0$, the rescaled variable $\mathcal{E}$ is distributed with the full Gaussian distribution

$$
P_{\text {cano }}^{(\mu<0)}(\mathcal{E})=\frac{1}{\sqrt{\pi}} e^{-\mathcal{E}^{2}}
$$

whereas for $\mu=0$, the rescaled variable is distributed with the half Gaussian distribution

$$
P_{\text {cano }}^{\left(F_{0}=0\right)}(\mathcal{E})=\theta(\mathcal{E}>0) \frac{2}{\sqrt{\pi}} e^{-\mathcal{E}^{2}}
$$




\section{Distribution of $E_{L}$ in the microcanonical ensemble}

In the presence of the microcanonical constraint (65), which fixes the end-point value of the potential $U(L)=F_{0} L$, we need the distribution of $E$ for the trajectories having exactly $U=F_{0} L$, i.e. we obtain from the joint distribution (26) with the notation $F_{0}=\mu \sigma$

$$
\left[P_{L}^{(\mu)}(E)\right]_{\text {micro }}=\frac{P_{L}^{\left(F_{0}=\mu \sigma\right)}(U=0, E)}{\int d E P_{L}^{\left(F_{0}=\mu \sigma\right)}(U=0, E)}=\theta(E>0) \theta(E>-\mu \sigma L)\left(\mu+\frac{2 E}{\sigma L}\right) e^{-\mu E} e^{-\frac{E^{2}}{\sigma L}}
$$

which exactly coincides with the formula (11) in [2] with the correspondence $\mu=-2 \bar{\delta}$ and $l=\sigma L$. We now consider the limit $L \rightarrow \infty$ with $\mu$ fixed, to compare with the results of the canonical ensemble.

For $\mu>0$, there exists a limit distribution that exactly coincides with (29)

$$
\mathcal{P}_{\text {micro }}^{(\mu>0)}(E) \underset{L \rightarrow \infty}{\simeq} \theta(E>0) \mu e^{-\mu E}
$$

At the critical point $\mu=0$, the appropriate rescaled variable is again $\mathcal{E}=E / \sqrt{4 \sigma L}$, but the corresponding distribution

$$
P_{\text {micro }}^{(\mu=0)}(\mathcal{E})=\theta(\mathcal{E}>0) 8 \mathcal{E} e^{-4 \mathcal{E}^{2}}
$$

does not coincide with the canonical result (32). In particular, it vanishes linearly as $\mathcal{E} \rightarrow 0$, in contrast with the canonical result which presents a finite density at the origin.

For $\mu<0$, the appropriate rescaled variable is not $\mathcal{E}$ in contrast with the canonical case, but it is

$$
v \equiv E-|\mu| \sigma L
$$

which is asymptotically distributed with the exponential distribution

$$
P_{\text {micro }}^{(\mu<0)}(v)=\theta(v>0) \mu e^{-\mu E}
$$

which is exactly the distribution of barriers against the bias (29 34) : this shows that the statistics for the barrier $E=U(0)-U\left(x_{0}\right)$ is actually completely determined in this case by the statistics of the barrier $v=U(L)-U\left(x_{0}\right)$ distributed with (37), since they are completely correlated via the microcanonical constraint $U(L)-U(0)=v-E=$ $F_{0} L$ (65).

\section{Discussion}

We first have to discuss the validity of the saddle-point method. At the critical point $\mu=0$, where the random variable $E_{L}$ scales as $\sqrt{L}$, and in the phase $\mu<0$, where the random variable $E_{L}$ is of order $L$, the saddle-point method is thus well justified at large $L$, at least for typical samples. On the contrary, in the phase $\mu>0$, where the random variable $E_{L}$ remains finite, the saddle-point method will be a good approximation only if $E$ is large, i.e. in the limit $\mu \rightarrow 0$ near the critical point.

On the other hand, since whenever the saddle-point analysis is valid, the variable $E_{L}$ is large, we may safely approximate the surface magnetization by

$$
\ln m_{1}^{s}=\ln \left(1+Z_{L}\right)^{-1 / 2} \underset{Z_{L}>>1}{\simeq}-(1 / 2) \ln Z_{L} \sim-E_{L} / 2
$$

and thus the results derived above for the distribution of $E_{L}$ indeed represents the probability distribution of the log of the surface magnetization. In particular, in the vicinity of the critical point, if one introduces the scaling variables $[2,7]$

$$
\begin{aligned}
& w=\frac{E}{\sqrt{\sigma L}}=\frac{-2 \ln m_{1}^{s}}{\sqrt{\sigma L}} \\
& \gamma=\mu \sqrt{\sigma L}
\end{aligned}
$$

we may write the following finite-size scaling forms for the probability distribution of $\left(-\ln m_{1}^{s}\right)$

$$
P_{L}\left(-\ln m_{1}^{s}\right)=\frac{2}{\sqrt{\sigma L}} Q\left(w=\frac{-2 \ln m_{1}^{s}}{\sqrt{\sigma L}} ; \gamma=\mu \sqrt{\sigma L}\right)
$$


where the scaling functions respectively read for the two ensembles (2833)

$$
\begin{aligned}
Q_{\text {cano }}(w ; \gamma) & =\theta(w>0)\left[\frac{1}{\sqrt{\pi}} e^{-\frac{(w+\gamma)^{2}}{4}}+\gamma e^{-\gamma w} \int_{\frac{w-\gamma}{2}}^{+\infty} \frac{d z}{\sqrt{\pi}} e^{-z^{2}}\right] \\
Q_{\text {micro }}(w ; \gamma) & =\theta(w>0) \theta(w>-\gamma)(2 w+\gamma) e^{-\gamma w-w^{2}}
\end{aligned}
$$

As emphasized in [2], the critical exponents are the same in the two ensembles (the scaling variables $(w, \gamma)$ are the same), but the finite-size scaling functions are different in the two ensembles.

We now turn to the question of the mean surface magnetization. As stressed in [1, 2], since the typical surface magnetization decays in the critical region as

$$
m_{1}^{s}=e^{-\frac{\sqrt{\sigma L}}{2} w}
$$

where $w$ is a random variable of order one, the mean surface magnetization will be governed by the rare samples that presents an anomalously big surface magnetization of order one, and its decay with $L$ will be governed by the measure of these rare samples as a function of $L$. However, since the limit distributions above are a priori not expected to describe well the tail $w \sim 1 / \sqrt{L}$ outside the scaling region, and since the approximations (38) are not valid anymore for $Z_{L} \sim 1$, it is necessary to discuss separately the samples having $Z_{L} \sim 1$ from the starting point (24) of the saddlepoint analysis. For these samples, the minimum $\left(-E_{L}\right)$ of the potential $U(x)$ on the interval $[0, L]$ has to remain of order one. At the critical point $\mu=0$, the decay of the mean surface magnetization in the canonical ensemble [1, 2]

$$
\left[m_{1}^{s}\right]_{\text {cano }} \underset{L \rightarrow \infty}{\propto} \frac{1}{\sqrt{L}}
$$

corresponds to the scaling of the probability for a Brownian path to remain above its starting point during a length $L$. In the microcanonical ensemble, the presence of the different decay [1, 2]

$$
\left[m_{1}^{s}\right]_{\text {micro }} \underset{L \rightarrow \infty}{\propto} \frac{1}{L}
$$

can be understood as the ratio between (i) the scaling as $1 / L^{3 / 2}$ for the probability of the first return to the origin after a length $L$, (ii) the scaling as $1 / \sqrt{L}$ for the probability to be at the origin at $L$. These two scalings, reflecting the measure of the rare samples having $Z_{L} \sim 1$, can actually be recovered from the scaling functions (43) as computed in [2], but the numerical prefactors given in equations (12-13) of [2] should not be considered as exact. Actually these prefactors are not expected to be universal, as was discussed for other quantities such as the average end-to-end correlation in [13].

In conclusion of this Section, we have shown that the limit distributions for the log of the surface magnetization obtained in 2] can be derived via a saddle point method in each sample, and have thus a very simple probabilistic interpretation : they describe the probability distribution of the minimum of a finite-size biased random walk, with different boundary conditions in the canonical and in the microcanonical ensembles. These limit distributions for the $\log$ of Kesten random variables should be considered as the analog of the Central-limit theorem for the log of a product of random variables [30] : they describe well typical samples and are expected to be universal. However, the computation of some averaged quantities which are dominated by rare events cannot be computed exactly from these limit distributions in the scaling regime. In the two next Section, we thus compute exactly the average surface magnetization in the two ensembles.

\section{EXACT RESULTS FOR THE SURFACE MAGNETIZATION IN THE CANONICAL ENSEMBLE}

\section{A. Exact expression for the averaged surface magnetization}

The probability distribution of the random variable $Z_{L}$ (15) in the case where the process $\{F(x)\}$ is a Brownian motion (21) has been already much studied by various methods [25, 31, 32]. Here, the most convenient starting point is the following exact result for its Laplace transform derived in [25]

$$
\begin{aligned}
{\left[e^{-p Z_{L}}\right]_{\text {cano }}=} & \sum_{0 \leq n<\frac{\mu}{2}} e^{-\sigma \operatorname{Ln}(\mu-n)} \frac{2(\mu-2 n)}{\Gamma(1+n) \Gamma(1+\mu-n)}\left(\frac{p}{\sigma}\right)^{\frac{\mu}{2}} K_{\mu-2 n}\left(2 \sqrt{\frac{p}{\sigma}}\right) \\
& +\frac{1}{2 \pi^{2}} \int_{0}^{+\infty} d q e^{-\frac{\sigma L}{4}\left(\mu^{2}+q^{2}\right)} q \sinh \pi q\left|\Gamma\left(-\frac{\mu}{2}+i \frac{q}{2}\right)\right|^{2}\left(\frac{p}{\sigma}\right)^{\frac{\mu}{2}} K_{i q}\left(2 \sqrt{\frac{p}{\sigma}}\right)
\end{aligned}
$$


where $\mu$ is the exponent (22), and where $\sigma$ represents the strength of the disorder (211). We refer the interested reader to the previous studies [25, 31] for a detailed discussion of this result. Here, we are interested into the surface magnetization $m_{1}^{s}$ which can be computed from the result (47) via the identity

$$
\left[m_{1}^{s}\right]_{\text {cano }}=\left[\left(1+Z_{L}\right)^{-1 / 2}\right]_{\text {cano }}=\frac{1}{\sqrt{\pi}} \int_{0}^{+\infty} d p p^{-1 / 2} e^{-p}\left[e^{-p Z_{L}}\right]_{\text {cano }}
$$

which yields in terms of the the Whittaker function (B3)

$$
\begin{aligned}
& {\left[m_{1}^{s}\right]_{\text {cano }}=\frac{\sigma^{\frac{1}{2}}}{\sqrt{\pi}} \sum_{0 \leq n<\frac{\mu}{2}} e^{-\sigma \operatorname{Ln}(\mu-n)}(\mu-2 n) \frac{\Gamma\left(n+\frac{1}{2}\right) \Gamma\left(\mu+\frac{1}{2}-n\right)}{\Gamma(1+n) \Gamma(1+\mu-n)}\left[\sigma^{-\frac{\mu}{2}} e^{\frac{1}{2 \sigma}} W_{-\frac{\mu}{2}, \frac{\mu}{2}-n}\left(\frac{1}{\sigma}\right)\right]} \\
& +\frac{\sigma^{\frac{1}{2}}}{4 \pi^{5 / 2}} \int_{0}^{+\infty} d q e^{-\frac{\sigma L}{4}\left(\mu^{2}+q^{2}\right)} q \sinh \pi q\left|\Gamma\left(-\frac{\mu}{2}+i \frac{q}{2}\right)\right|^{2}\left|\Gamma\left(\frac{\mu}{2}+i \frac{q}{2}+\frac{1}{2}\right)\right|^{2}\left[\sigma^{-\frac{\mu}{2}} e^{\frac{1}{2 \sigma}} W_{-\frac{\mu}{2}, i \frac{q}{2}}\left(\frac{1}{\sigma}\right)\right]
\end{aligned}
$$

As a comparison, the negative moment of order $(1 / 2)$ reads

$$
\begin{aligned}
{\left[Z_{L}^{-\frac{1}{2}}\right]_{\text {cano }} } & =\frac{\sigma^{\frac{1}{2}}}{\sqrt{\pi}} \sum_{0 \leq n<\frac{\mu}{2}} e^{-\sigma L n(\mu-n)}(\mu-2 n) \frac{\Gamma\left(\frac{1}{2}+n\right) \Gamma\left(\frac{1}{2}+\mu-n\right)}{\Gamma(1+n) \Gamma(1+\mu-n)} \\
& +\frac{\sigma^{\frac{1}{2}}}{4 \pi^{5 / 2}} \int_{0}^{+\infty} d q e^{-\frac{\sigma L}{4}\left(\mu^{2}+q^{2}\right)} q \sinh \pi q\left|\Gamma\left(-\frac{\mu}{2}+i \frac{q}{2}\right)\right|^{2}\left|\Gamma\left(\frac{1}{2}+\frac{\mu}{2}+i \frac{q}{2}\right)\right|^{2}
\end{aligned}
$$

i.e. the two results are very similar : the only differences are in the the factors inside [... in (49).

\section{B. Ordered phase $\mu=-2 \delta>0$}

For $\mu>0$, there exists a limit distribution in the large $L$ limit, whose generating function is simply given by the term $n=0$ in (47)

$$
\left[e^{-p Z_{\infty}}\right]_{\text {cano }}=\frac{2}{\Gamma(\mu)}\left(\frac{p}{\sigma}\right)^{\frac{\mu}{2}} K_{\mu}\left(2 \sqrt{\frac{p}{\sigma}}\right)
$$

It corresponds to the probability distribution 22, 25]

$$
\mathcal{P}\left(Z_{\infty}\right)=\frac{\sigma}{\Gamma(\mu)}\left(\frac{1}{\sigma Z_{\infty}}\right)^{1+\mu} e^{-\frac{1}{\sigma Z_{\infty}}}
$$

For the RTFIC, the case $\mu>0$ indeed corresponds to the ordered phase $\delta<0$ where the surface magnetization remains finite in the thermodynamic limit

$$
\left[m_{s}(\infty)\right]_{\text {cano }}(\mu>0)=\mu \sqrt{\sigma} \frac{\left(\Gamma\left(\frac{1}{2}+\mu\right)\right)}{\Gamma(1+\mu)}\left[\sigma^{\frac{-\mu}{2}} e^{\frac{1}{2 \sigma}} W_{-\frac{\mu}{2}, \frac{\mu}{2}}\left(\frac{1}{\sigma}\right)\right]
$$

As a comparison, we have

$$
\left[Z_{\infty}^{-\frac{1}{2}}\right]_{\text {cano }}=\mu \sqrt{\sigma} \frac{\Gamma\left(\frac{1}{2}+\mu\right)}{\Gamma(1+\mu)}
$$

\section{Critical point $\mu=0$}

At the critical point $\mu=0$, the result (47) for the Laplace transform of the probability distribution of $Z_{L}$ simplifies into

$$
\left[e^{-p Z_{L}}\right]_{\text {cano }}=\frac{2}{\pi} \int_{0}^{+\infty} d q e^{-\frac{\sigma L}{4} q^{2}} \cosh \pi \frac{q}{2} K_{i q}\left(2 \sqrt{\frac{p}{\sigma}}\right)
$$


and the result (49) for the average surface magnetization becomes using (B4)

$$
\begin{aligned}
{\left[m_{1}^{s}\right]_{\text {cano }}(\mu=0) } & =\frac{e^{\frac{1}{2 \sigma}}}{\pi} \int_{0}^{+\infty} d q e^{-\frac{\sigma L}{4} q^{2}} K_{i \frac{q}{2}}\left(\frac{1}{2 \sigma}\right) \\
& =\frac{e^{\frac{1}{2 \sigma}}}{\sqrt{\pi \sigma L}} K_{0}\left(\frac{1}{2 \sigma}\right)\left[1-\frac{K_{0}^{\prime \prime}\left(\frac{1}{2 \sigma}\right)}{4 \sigma L K_{0}\left(\frac{1}{2 \sigma}\right)}+O\left(1 / L^{3}\right)\right]
\end{aligned}
$$

whereas the negative moment of order $(1 / 2)$ reads

$$
\left[Z_{L}^{-\frac{1}{2}}\right]_{\text {cano }}(\mu=0)=\frac{1}{\sqrt{L}}
$$

Both results are indeed dominated by rare configurations of measure $1 / \sqrt{L}$ which have a $Z_{L}$ of order 1 , in agreement with the previous studies [1, 2]. The different prefactors in the leading terms of (5758) can be understood via the series expansion

$$
\left(1+Z_{L}\right)^{-1 / 2}=Z_{L}^{-1 / 2}-(1 / 2) Z_{L}^{-3 / 2}+\ldots
$$

Since the averages are dominated by the rare events where $Z_{L}$ is of order 1 with probability of order $1 / \sqrt{L}$, all the terms in the series expansion will contribute to the prefactor found in (157).

It is actually interesting to consider the distribution of $Z_{L}$ among these rare events : the Laplace transform has for leading term as $L \rightarrow \infty$ with $p$ fixed

$$
\left[e^{-p Z_{L}}\right]_{\text {cano }} \underset{L \rightarrow \infty}{\simeq} \frac{2}{\sqrt{\pi \sigma L}} K_{0}\left(2 \sqrt{\frac{p}{\sigma}}\right)+O\left(\frac{1}{L^{3 / 2}}\right)
$$

which corresponds after Laplace inversion to the following behavior for the probability distribution $P_{L}(z)$ at large $L$ with fixed $z$

$$
P_{L}^{c a n o}(z) \underset{L \rightarrow \infty}{\simeq} \frac{1}{\sqrt{\pi \sigma L}} \frac{e^{-\frac{1}{\sigma z}}}{z}+O\left(\frac{1}{L^{3 / 2}}\right)
$$

This result describes the tail of rare events with $z$ fixed outside the scaling region $\ln z \sim \sqrt{L}$ studied in Section

\section{Finite-size scaling function in the critical region}

In the critical region parameterized by the rescaled parameter $\gamma$ (40), we obtain that the distribution of $Z_{L}$ of order one is characterized by the Laplace transform

$$
\left[e^{-p Z_{L}}\right]_{\text {cano }}(\gamma)=\frac{2}{\sqrt{\pi \sigma L}} K_{0}\left(2 \sqrt{\frac{p}{\sigma}}\right)\left[\theta(\gamma>0) \gamma \sqrt{\pi}+e^{-\frac{\gamma^{2}}{4}}-|\gamma| \int_{\frac{|\gamma|}{2}}^{+\infty} d v e^{-v^{2}}\right]+O\left(\frac{1}{L}\right)
$$

where the term containing the theta function comes from the bound state $n=0$, and the other terms from the continuum. Actually, we may rewrite for arbitrary sign of $\gamma$

$$
\left[e^{-p Z_{L}}\right]_{\text {cano }}(\gamma)=\frac{2}{\sqrt{\pi \sigma L}} K_{0}\left(2 \sqrt{\frac{p}{\sigma}}\right)\left[e^{-\frac{\gamma^{2}}{4}}+\gamma \int_{-\frac{\gamma}{2}}^{+\infty} d v e^{-v^{2}}\right]+O\left(\frac{1}{L}\right)
$$

This result is thus completely factorized into (i) the distribution of $z$ among the rare events at the critical point (60) times (ii) the function of $\gamma$ inside [..] that coincides with eq (12) of [2] and that represents the probability of $E_{L}=0$ in the scaling function discussed in previous Section III] As a consequence, the finite-size scaling form for the averaged magnetization reads

$$
\left[m_{1}^{s}\right]_{\text {cano }}(\gamma) \underset{L \rightarrow \infty}{\stackrel{\simeq}{\rightarrow}} \frac{c_{m}}{\sqrt{\pi \sigma L}}\left[e^{-\frac{\gamma^{2}}{4}}+\gamma \int_{-\frac{\gamma}{2}}^{+\infty} d v e^{-v^{2}}\right]+O\left(\frac{1}{L}\right)
$$

where $c_{m}$ is a non-universal constant, found here to be $c_{m}=e^{\frac{1}{2 \sigma}} K_{0}\left(\frac{1}{2 \sigma}\right)$ (57), and where all dependence in $\gamma$ corresponds to the prediction of the scaling regime as derived in [2]. We now compute the same observables for the microcanonical ensemble. 


\section{EXACT RESULTS FOR THE SURFACE MAGNETIZATION IN THE MICROCANONICAL ENSEMBLE}

In this Section, we derive exact results for the continuous Kesten variable with a Gaussian disorder in the presence of the microcanonical constraint

$$
\int_{0}^{L} d y F(y)=F_{0} L=\mu \sigma L
$$

and we compare with the results of the canonical ensemble given in the previous Section.

\section{A. Probability distribution}

The Laplace transform of the probability distribution of $Z_{L}$ in the microcanonical ensemble may be written as follows in terms of path-integrals

$$
\left[e^{-p Z_{L}}\right]_{\text {micro }}=\frac{\int \mathcal{D} F(x) e^{-\frac{1}{4 \sigma} \int_{0}^{L}\left[F(x)-F_{0}\right]^{2}-p \int_{0}^{L} d x e^{-\int_{0}^{x} d y F(y)} \delta\left(\int_{0}^{L} d x F(x)-F_{0} L\right)}}{\int \mathcal{D} F(x) e^{-\frac{1}{4 \sigma} \int_{0}^{L}\left[F(x)-F_{0}\right]^{2}} \delta\left(\int_{0}^{L} d x F(x)-F_{0} L\right)}
$$

where the delta function represents the microcanonical constraint (65). Rewriting this path-integral over the force $F(x)$ as a path-integral over the potential $U(x)=\int_{0}^{x} d y F(y)$ as in [25], we obtain

$$
\left[e^{-p Z_{L}}\right]_{\text {micro }}=\frac{\int_{U(0)=0}^{U(L)=F_{0} L} \mathcal{D} U(x) e^{-\frac{1}{4 \sigma} \int_{0}^{L}\left(\frac{d U(x)}{d x}\right)^{2}-p \int_{0}^{L} d x e^{-U(x)}}}{\int_{U(0)=0}^{U(L)=F_{0} L} \mathcal{D} U(x) e^{-\frac{1}{4 \sigma} \int_{0}^{L}\left(\frac{d U(x)}{d x}\right)^{2}}}
$$

The denominator is a simple Gaussian propagator

$$
\int_{U(0)=0}^{U(L)=F_{0} L} \mathcal{D} U(x) e^{-\frac{1}{4 \sigma} \int_{0}^{L}\left(\frac{d U(x)}{d x}\right)^{2}}=\frac{1}{\sqrt{4 \pi \sigma L}} e^{-\frac{(U(L)-U(0))^{2}}{4 \sigma L}}=\frac{1}{\sqrt{4 \pi \sigma L}} e^{-\frac{F_{0}^{2}}{4 \sigma} L}
$$

The path-integral in the numerator is equivalent to a propagator of quantum mechanics corresponding to the Hamiltonian $H=-\sigma d^{2} / d U^{2}+p e^{-U}$. Expanding this path-integral into the eigenstates $\psi_{k}(U)$ of the Hamiltonian as described in [25], we finally get with $F_{0}=\mu \sigma$

$$
\begin{aligned}
{\left[e^{-p Z_{L}}\right]_{\text {micro }} } & =\sqrt{4 \pi \sigma L} e^{\frac{\mu^{2}}{4} \sigma L} \int_{-\infty}^{+\infty} \frac{d k}{2 \pi} e^{-k^{2} L} \psi_{k}^{*}(0) \psi_{k}(\mu \sigma L) \\
& =\frac{2}{\pi^{3 / 2}} \sqrt{\sigma L} e^{\frac{\mu^{2}}{4} \sigma L} \int_{0}^{+\infty} d q e^{-\frac{q^{2}}{4} \sigma L} q \sinh \pi q K_{i q}\left(2 \sqrt{\frac{p}{\sigma}}\right) K_{i q}\left(2 \sqrt{\frac{p}{\sigma}} e^{-\frac{\mu \sigma L}{2}}\right)
\end{aligned}
$$

which should be compared with (47).

\section{B. Surface magnetization}

The surface magnetization $m_{1}^{s}$ which can then be computed via the identity

$$
\left[m_{1}^{s}\right]_{\text {micro }}=\left[\left(1+Z_{L}\right)^{-1 / 2}\right]_{\text {micro }}=\frac{1}{\sqrt{\pi}} \int_{0}^{+\infty} d p p^{-1 / 2} e^{-p}\left[e^{-p Z_{L}}\right]_{\text {micro }}
$$

which yields

$$
\left[m_{1}^{s}\right]_{\text {micro }}=\frac{2}{\pi^{2}} \sqrt{\sigma L} e^{\frac{\mu^{2}}{4} \sigma L} \int_{0}^{+\infty} d q e^{-\frac{q^{2}}{4} \sigma L} q \sinh \pi q \int_{0}^{+\infty} d p p^{-1 / 2} e^{-p} K_{i q}\left(2 \sqrt{\frac{p}{\sigma}}\right) K_{i q}\left(2 \sqrt{\frac{p}{\sigma}} e^{-\frac{\mu \sigma L}{2}}\right)
$$




\section{Ordered phase $\mu=-2 \delta>0$}

For $\mu>0$, we have seen in the canonical ensemble that there exists a limit distribution in the large $L$ limit, given by (51). To recover this result in the microcanonical ensemble, we may use (B1) at lowest order to obtain

$$
K_{i q}\left(2 \sqrt{\frac{p}{\sigma}} e^{-\frac{\mu \sigma L}{2}}\right)=\frac{\pi}{2 i \sinh \pi q}\left[\frac{\left(\frac{p}{\sigma}\right)^{-i \frac{q}{2}}}{\Gamma(1-i q)} e^{i q \frac{\mu \sigma L}{2}}-\frac{\left(\frac{p}{\sigma}\right)^{i \frac{q}{2}}}{\Gamma(1+i q)} e^{-i q \frac{\mu \sigma L}{2}}\right]+O\left(e^{-\mu \sigma L}\right)
$$

which yields

$$
\begin{aligned}
{\left[e^{-p Z_{\infty}}\right]_{\text {micro }} } & =\lim _{L \rightarrow \infty}\left(\frac{\sqrt{\sigma L}}{2 i \pi^{1 / 2}} \int_{-\infty}^{+\infty} d q q K_{i q}\left(2 \sqrt{\frac{p}{\sigma}}\right)\left[\frac{\left(\frac{p}{\sigma}\right)^{-i \frac{q}{2}}}{\Gamma(1-i q)} e^{-\frac{(q-i \mu)^{2}}{4} \sigma L}-\frac{\left(\frac{p}{\sigma}\right)^{i \frac{q}{2}}}{\Gamma(1+i q)} e^{-\frac{(q+i \mu)^{2}}{4} \sigma L}\right]\right) \\
& =\frac{2}{\Gamma(\mu)}\left(\frac{p}{\sigma}\right)^{\frac{\mu}{2}} K_{\mu}\left(2 \sqrt{\frac{p}{\sigma}}\right)
\end{aligned}
$$

where the final limit is obtained by taking into account the saddles in the complex plane at $q= \pm i \mu$ respectively for the two terms. The limit distribution in the thermodynamic limit is thus the same as in the canonical ensemble (51).

\section{Critical point $\mu=0$}

At the critical point $\mu=0$, the probability distribution of $Z_{L}$ is characterized by the Laplace transform

$$
\left[e^{-p Z_{L}}\right]_{\text {micro }}(\mu=0)=\frac{2}{\pi^{3 / 2}} \sqrt{\sigma L} \int_{0}^{+\infty} d q e^{-\frac{\sigma L}{4} q^{2}} q \sinh \pi q K_{i q}^{2}\left(2 \sqrt{\frac{p}{\sigma}}\right)
$$

which should be compared with (55). The surface magnetization (71) reads

$$
\begin{aligned}
{\left[m_{1}^{s}\right]_{\text {micro }}=} & \frac{2}{\pi^{2}} \sqrt{\sigma L} \int_{0}^{+\infty} d q e^{-\frac{q^{2}}{4} \sigma L} q \sinh \pi q \int_{0}^{+\infty} d p p^{-1 / 2} e^{-p} K_{i q}^{2}\left(2 \sqrt{\frac{p}{\sigma}}\right) \\
& =\frac{8 \pi}{\sigma L} \int_{0}^{+\infty} d p p^{-1 / 2} e^{-p} K_{0}^{2}\left(2 \sqrt{\frac{p}{\sigma}}\right)+O\left(\frac{1}{L^{2}}\right)
\end{aligned}
$$

As a comparison, the negative moment of order $(1 / 2)$ reads

$$
\left[Z_{L}^{-1 / 2}\right]_{\text {micro }}(\mu=0)=\pi \sigma \sqrt{L} \int_{0}^{+\infty} d q e^{-q^{2} \frac{\sigma L}{4}} q \tanh (\pi q)=\frac{2 \pi^{5 / 2}}{L \sqrt{\sigma}}\left[1-\frac{2 \pi^{2}}{\sigma L}+O\left(1 / L^{2}\right)\right]
$$

Both are indeed dominated by the rare events where $Z_{L} \sim 1$, which have a measure of order $1 / L$ in the microcanonical ensemble, in agreement with previous studies [1, 2].

It is now interesting to consider the distribution of $Z_{L}$ for these rare events : the Laplace transform has for leading term as $L \rightarrow \infty$ with $p$ fixed

$$
\left[e^{-p Z_{L}}\right]_{\text {micro }}(\mu=0) \underset{L}{\simeq} \underset{\rightarrow \infty}{\simeq} \frac{4}{\sigma L} K_{0}^{2}\left(2 \sqrt{\frac{p}{\sigma}}\right)+O\left(\frac{1}{L^{2}}\right)
$$

This leading behavior is actually very simply related to the analog result (60) in the canonical ensemble

$$
\left[e^{-p Z_{L}}\right]_{\text {micro }}^{\text {rare }} \simeq \pi\left(\left[e^{-p Z_{L}}\right]_{\text {cano }}^{\text {rare }}\right)^{2}
$$

which corresponds after Laplace inversion to a convolution for the probability distribution $P_{L}^{\text {cano }}(z)$ at large $L$ with fixed $z$ (61)

$$
P_{L}^{\text {micro }}(z) \underset{L \rightarrow \infty}{\simeq} \frac{2}{\sigma L z} K_{0}\left(\frac{2}{\sigma z}\right) e^{-\frac{2}{\sigma z}}
$$

This result describes the tail of rare events with $z$ fixed in the microcanonical ensemble, outside the scaling region $\ln z \sim \sqrt{L}$ studied in Section III 


\section{E. Finite size scaling function in the critical region}

In the critical region inside the ordered phase $\mu=-2 \delta>0$, parameterized by the rescaled parameter $\gamma$ (40), the analog of (72) reads with $q=k / \sqrt{\sigma L}$

$$
K_{i q}\left(2 \sqrt{\frac{p}{\sigma}} e^{-\frac{\gamma \sqrt{\sigma L}}{2}}\right)=\frac{\pi}{2 i \sinh \pi q}\left[\frac{\left(\frac{p}{\sigma}\right)^{-i \frac{q}{2}}}{\Gamma(1-i q)} e^{i q \frac{\gamma \sqrt{\sigma L}}{2}}-\frac{\left(\frac{p}{\sigma}\right)^{i \frac{q}{2}}}{\Gamma(1+i q)} e^{-i q \frac{\gamma \sqrt{\sigma L}}{2}}\right]+O\left(e^{-\gamma \sqrt{\sigma L}}\right)
$$

which yields for the generating function with fixed $Z$ at large $L$

$$
\left[e^{-p Z_{L}}\right]_{\text {micro }}(\gamma=\mu \sqrt{\sigma L}>0)=\gamma \frac{2}{\sqrt{\sigma L}} K_{0}\left(2 \sqrt{\frac{p}{\sigma}}\right)+O\left(\frac{1}{L}\right)
$$

The finite-size scaling for the average surface magnetization is thus simply

$$
\left[m_{1}^{s}\right]_{\text {micro }}(\gamma>0) \underset{L \rightarrow \infty}{\simeq} \frac{\gamma c_{m}}{\sqrt{\sigma L}}
$$

that should be compared with the corresponding result in the canonical ensemble (64) : $c_{m}$ is the same constant, and the scaling function of $\gamma$ has been simply replaced by the factor $\gamma$ alone, that again corresponds to the prediction of the scaling regime as derived in [2]. Here, the amplitude of the leading term in $1 / \sqrt{L}$ vanishes at the critical point $\gamma=0$, as it should to recover the scaling $1 / L$ at the critical point.

In the critical region inside the disordered phase $\mu=-2 \delta<0$, parameterized by the rescaled parameter $\gamma$ (40), the argument in the Bessel function is now exponentially large in $\sqrt{L}$ instead of exponentially small as in (81). Taking into account the asymptotic behavior of Bessel function at large argument (B2) we thus obtain that $\gamma$ is not the appropriate scaling variable near the critical point on the disordered side. Instead, to obtain the finite-size scaling function, we need the scaling variable

$$
\rho \equiv \mu \sigma L
$$

that leads to

$$
\left[e^{-p Z_{L}}\right]_{\text {micro }}(\rho=\mu \sigma L<0) \underset{L}{\simeq} \frac{4}{\sigma L} K_{0}\left(2 \sqrt{\frac{p}{\sigma}}\right) K_{0}\left(2 \sqrt{\frac{p}{\sigma}} e^{-\frac{\rho}{2}}\right)+O\left(\frac{1}{L^{2}}\right)
$$

After Laplace inversion, the probability distribution $P_{L}(z)$ at large $L$ with fixed $z$ and $\rho$ is given by

$$
P_{L}^{\text {micro }}(z, \rho) \underset{L}{\stackrel{\sim}{\rightarrow}} \frac{1}{\sigma L z} \int_{0}^{1} \frac{d u}{u(1-u)} e^{-\frac{1}{\sigma z}\left(\frac{1}{u}+\frac{e^{-\rho}}{1-u}\right)}+O\left(\frac{1}{L^{2}}\right)
$$

So here, in contrast with the corresponding result (63) in the canonical ensemble, this result is not factorized into a function of $z$ times a function of $\rho$. The finite-size scaling form for the average surface magnetization is thus given by

$$
\left[m_{1}^{s}\right]_{\text {micro }}(\rho=\mu \sigma L<0) \underset{L}{\stackrel{\sim}{\rightarrow}} \frac{4}{\sqrt{\pi} \sigma L} \int_{0}^{+\infty} d p p^{-1 / 2} e^{-p} K_{0}\left(2 \sqrt{\frac{p}{\sigma}}\right) K_{0}\left(2 \sqrt{\frac{p}{\sigma}} e^{-\frac{\rho}{2}}\right)+O\left(\frac{1}{L^{2}}\right)
$$

In conclusion, the critical regime on the disordered side is thus governed by the scaling variable $\rho=\mu \sigma L<0$ in the microcanonical ensemble, whereas it is governed by the scaling variable $\gamma=\mu \sqrt{\sigma L}<0$ in the canonical ensemble. To understand these results, it is thus useful to recall the origin of the presence of two different correlation length exponents in the RTFIC.

\section{F. Discussion on the two correlation length exponents}

The presence of two different correlation length exponents $\nu=2$ and $\tilde{\nu}=1$ in the RTFIC has been discussed in detail in 7]. The definition of these two length scale for the Brownian process $(U(L)-U(0))$ can be summarized as follows [7] : the first length scale corresponds to the length $\tilde{\xi}$ where the mean value $<U(L)-U(0)>=F_{0} L=\mu \sigma L$ is of order one, which yields

$$
\tilde{\xi} \sim \frac{1}{\sigma \mu^{\tilde{\nu}}} \quad \text { with } \quad \tilde{\nu}=1
$$


The second length scale corresponds to the length $\xi$ where most of the samples indeed have $(U(L)-U(0))$ of the same sign of the mean value, i.e. the scale $\sqrt{\sigma L}$ of the fluctuations should be of the same order of the mean value, which yields

$$
\xi \sim \frac{1}{\sigma \mu^{\nu}} \quad \text { with } \quad \nu=2
$$

These two length scales appear in various quantities in the RTFIC. In particular, the averaged correlation is governed by the exponent $\nu=2$, whereas the typical correlation is governed by the exponent $\tilde{\nu}=1$ []. More generally, the presence of these two length-scales is also well known in related models, for instance in the eigenstates of the Fokker-Planck operator in a Sinai potential 22, 28] and in one-dimensional random-hopping Hamiltonian for fermions [33, 34].

In view of this knowledge, the interpretation of our results obtained in this Section for the averaged surface magnetization is as follows : in the canonical ensemble, the critical behavior is governed by the exponent $\nu=2$, whereas in the microcanonical ensemble, there are two different exponents in the two sides of the critical point : the ordered phase is governed by the exponent $\nu=2$, with an accidental vanishing of the amplitude at the critical point, as stressed in [2], but the disordered phase is governed by the exponent $\tilde{\nu}=1$, in agreement with the analysis given in 1 ] and in contrast with the interpretation given in [2]. More generally, the appearance of these two exponents for some given observable in the two ensembles should not be too surprising : indeed, the requirement that almost all samples of size $L$ indeed "know" the sign of $F_{0}$ involves the length-scale $\xi$ [89) in the canonical ensemble, whereas it involves

the length $\tilde{\xi}(88)$ in the microcanonical ensemble. So the very definitions of the two length scales $\xi$ and $\tilde{\xi}$ show that the microcanonical constraint can indeed play an important role for the critical exponents.

\section{G. Obtaining the exponent $\tilde{\nu}$ from the scaling regime}

As a final remark, it is now useful to discuss how the exponent $\tilde{\nu}=1$, found in this Section by an exact path-integral method for the averaged surface magnetization, can be understood from the knowledge of the scaling regime (43), that a priori only contains the exponent $\nu=2$. The evaluation of the averaged surface magnetization from the scaling function (43) in the disordered phase

$$
\left[m_{1}^{s}\right]_{\text {micro }}^{\text {scaling }}(\mu<0)=\int_{0}^{+\infty} d w Q_{\text {micro }}(w ; \gamma) e^{-\frac{\sqrt{\sigma L}}{2} w}
$$

shows that the origin of the "anomalous" exponent $\tilde{\nu}=1$ can be traced back to the presence of the theta function $\theta(w>-\gamma)$ in the scaling function $Q_{\text {micro }}(w ; \gamma)$, i.e. the probability density $Q_{\text {micro }}(w ; \gamma)$ vanishes at a finite positive value in $w$ in the disordered phase. This remark will be useful in the next Sections on more complicated observables, where we will compute the probability distributions in the scaling regime, and where direct methods to compute exactly the averaged values such as the path-integral method used in this Section will not be available.

\section{CORRELATION BETWEEN THE TWO SURFACE MAGNETIZATIONS IN THE TWO ENSEMBLES}

In this Section, we consider the correlation $\left(m_{1}^{s} m_{L}^{s}\right)$ (6) between the two surface magnetizations that has been numerically studied in [2]. We compute its universal limit distributions at large $L$ in the two ensembles.

\section{A. Saddle-point method in each sample}

The correlation $\left(m_{1}^{s} m_{L}^{s}\right)$ can be expressed in closed form in an arbitrary sample as the surface magnetization (7)

$$
m_{1}^{s} m_{L}^{s}=\left[1+Z_{L}\right]^{-1 / 2}\left[1+\tilde{Z}_{L}\right]^{-1 / 2}
$$


where $Z_{L}$ and $\tilde{Z}_{L}$ are two Kesten variables defined in terms of the same random potential $U(x)=\int_{0}^{x} F(y) d y$ in the continuous version (15)

$$
\begin{aligned}
& Z_{L}=\int_{0}^{L} d x e^{U(0)-U(x)} \\
& \tilde{Z}_{L}=\int_{0}^{L} d x e^{U(x)-U(L)}
\end{aligned}
$$

The saddle-point method in a given sample (24) on the associated random potential $U(x)=\int_{0}^{x} d y F(y)$ leads to

$$
\begin{aligned}
Z_{L} & \underset{L}{\stackrel{\sim}{\rightarrow}} e^{U(0)-U_{\min }} Z_{\text {valley }} \\
\tilde{Z}_{L} & \underset{L}{\stackrel{\sim}{\rightarrow}} e^{U_{\max }-U(L)} \tilde{Z}_{\text {valley }}
\end{aligned}
$$

in terms of the minimum $U_{\min }$ and maximum $U_{\max }$ reached by the process $U(x)$ on the interval $[0, L]$. Since both Kesten variables are typically large in the critical region, we may replace as in (38)

$$
\ln \left(m_{1}^{s} m_{L}^{s}\right) \underset{L \rightarrow \infty}{\simeq}-\frac{1}{2} \ln Z_{L}-\frac{1}{2} \tilde{Z}_{L} \underset{L \rightarrow \infty}{\simeq}-\frac{U(0)-U_{\min }+U_{\max }-U(L)}{2}
$$

so the limit distribution of $\ln \left(m_{1}^{s} m_{L}^{s}\right)$ can be computed in terms of the distribution of the random variable

$$
D_{L} \equiv A(L)-U(L)
$$

where $A(L)$ is the amplitude of the Brownian trajectory for $0 \leq x \leq L$

$$
A(L) \equiv U_{\max }-U_{\min }
$$

and where $U(0)=0$ is the starting point and $U(L)$ the final point.

\section{B. Joint distribution of the amplitude $A$ and the end-point $U$}

The joint probability $P_{L}^{(\mu)}(A, U)$ of the amplitude $A_{L}=U_{\max }-U_{\min }=U_{b}-U_{a}$ and the end-point $U$ when starting at $U_{0}=0$ has for Laplace transform with respect to $L$ (see Appendix

$$
\begin{aligned}
& \hat{P}^{(\mu)}(A, U ; p) \equiv \int_{0}^{+\infty} d L e^{-p L} P_{L}^{(\mu)}(A, U) \\
& =\theta(A>|U|) e^{\frac{\mu}{2} U} \frac{\cosh q U(q(A-|U|) \operatorname{coth} q A-1)+\sinh q|U| \operatorname{coth} q A}{\sigma \sinh ^{2} q A}
\end{aligned}
$$

where

$$
q \equiv \sqrt{\frac{p}{\sigma}+\frac{\mu^{2}}{4}}
$$

The Laplace inversion yields

$$
\begin{aligned}
P_{L}^{(\mu)}(A, U) & =\theta(A>|U|) \frac{e^{-\frac{(U-\mu \sigma L)^{2}}{4 \sigma L}}}{2 \sqrt{\pi}(\sigma L)^{3 / 2}} \sum_{k=-\infty}^{+\infty} e^{-k^{2} \frac{A^{2}}{\sigma L}}\left[(A-|U|) k^{2}\left(\frac{(U-2 k A)^{2}}{\sigma L}-2\right) e^{k \frac{A U}{\sigma L}}\right. \\
& -2 k(k-1)(|U|-2 k A) e^{\left.k \frac{A|U|}{\sigma L}\right]}
\end{aligned}
$$

We now discuss the microcanonical ensemble and the canonical ensemble respectively. 


\section{Microcanonical ensemble}

In the microcanonical ensemble with the constraint $U(L)=\mu \sigma L$, the probability distribution of the amplitude $A_{L}=U_{\max }-U_{\min }=U_{b}-U_{a}$ reads (101)

$$
\begin{aligned}
{\left[P_{L}^{(\mu)}(A)\right]_{\text {micro }} } & =\frac{P_{L}^{(\mu)}(A, \mu \sigma L)}{\int d A P_{L}^{(\mu)}(A, \mu \sigma L)}=\theta(A>|\mu| \sigma L) \frac{1}{(\sigma L)} \sum_{k=-\infty}^{+\infty} e^{-k^{2} \frac{A^{2}}{\sigma L}} \\
& {\left[(A-|\mu| \sigma L) k^{2}\left(\frac{(\mu \sigma L-2 k A)^{2}}{\sigma L}-2\right) e^{k A \mu}-2 k(k-1)(|\mu| \sigma L-2 k A) e^{k A|\mu|}\right] }
\end{aligned}
$$

Since $D=A-\mu \sigma L$, we may now write the final results for the various cases $\mu=0, \mu>0$ and $\mu<0$.

$$
\text { 1. Critical case } \mu=0
$$

In the critical case, the probability distribution of the variable $D=A$ reads

$$
\left[P_{L}^{(\mu=0)}(D)\right]_{\text {micro }}=\theta(D>0) \frac{4 D}{\sigma L} \sum_{k=1}^{+\infty} k^{2}\left(2 k^{2} \frac{D^{2}}{\sigma L}-3\right) e^{-k^{2} \frac{D^{2}}{\sigma L}}
$$

We thus obtain that the distribution of the variable $\ln \left(m_{1}^{s} m_{L}^{s}\right)$ (96) takes the scaling form

$$
\left[\mathcal{P}_{L}\left(-\ln \left(m_{1}^{s} m_{L}^{s}\right)\right)\right]_{\text {micro }}=\frac{2}{\sqrt{\sigma L}} \mathcal{P}_{\text {micro }}^{(0)}\left(\mathcal{D}=\frac{-2 \ln \left(m_{1}^{s} m_{L}^{s}\right)}{\sqrt{\sigma L}}\right)
$$

with the scaling function

$$
\begin{aligned}
P_{\text {micro }}^{(0)}(\mathcal{D}) & =\theta(\mathcal{D}>0) 4 \mathcal{D} \sum_{k=1}^{+\infty} k^{2}\left(2 k^{2} \mathcal{D}^{2}-3\right) e^{-k^{2} \mathcal{D}^{2}} \\
& =\theta(\mathcal{D}>0) \frac{4 \sqrt{\pi}}{\mathcal{D}^{4}} \sum_{n=1}^{+\infty} n^{2} \pi^{2}\left(\frac{2 n^{2} \pi^{2}}{\mathcal{D}^{2}}-3\right) e^{-n^{2} \frac{\pi^{2}}{\mathcal{D}^{2}}}
\end{aligned}
$$

These two series representations are useful to study the asymptotic behaviors at small and large arguments respectively. The decay at large $\mathcal{D}$ is given by

$$
P_{\text {micro }}(\mathcal{D}) \underset{D \rightarrow \infty}{\simeq} 8 \mathcal{D}^{3} e^{-\mathcal{D}^{2}}
$$

and at the origin, there is an essential singularity

$$
P_{\text {micro }}(\mathcal{D}) \underset{D \rightarrow 0}{\simeq} \frac{8 \pi^{9 / 2}}{\mathcal{D}^{6}} e^{-\frac{\pi^{2}}{\mathcal{D}^{2}}}
$$

This behavior of the scaling function at small argument yields the following dependence in $L$ for the averaged correlation of the two surface magnetizations

$$
\left[m_{1}^{s} m_{L}^{s}\right]_{\text {micro }}^{\text {scaling }} \equiv \int_{0}^{+\infty} d \mathcal{D} P_{\text {micro }}(\mathcal{D}) e^{-\mathcal{D} \frac{\sqrt{\sigma L}}{2}} \underset{L \rightarrow \infty}{\propto} L^{2 / 3} e^{-\frac{3}{2}\left(\frac{\pi^{2}}{2} \sigma L\right)^{1 / 3}}
$$

that should be compared with the numerical fits discussed in [2].

\section{2. $\quad$ Ordered phase $\mu>0$}

In the ordered phase $\mu>0$, there exists a limit probability distribution for $D$ as $L \rightarrow \infty$

$$
\left[P_{\infty}^{(\mu>0)}(D)\right]_{\text {micro }}=\theta(D>0) \mu^{2} D e^{-\mu D}
$$

This result corresponds to the convolution of two independent barriers against the bias as it should. 


\section{Disordered phase $\mu<0$}

For $\mu<0$, the minimal value of $D$ is $D_{\min }=-2 \mu \sigma L$, so it is convenient to set

$$
D \equiv 2|\mu| \sigma L+W
$$

and there exists a limit distribution for $W$ in the large $L$ limit that reads

$$
\left[P_{L \rightarrow \infty}^{(\mu<0)}(W)\right]_{\text {micro }}=\theta(W>0) \mu^{2} W e^{-|W|}
$$

This results simply represents the distribution of the sum of two barriers against the bias : the comparison with the result (37) concerning one surface magnetization shows that it can be interpreted in the same way.

\section{Finite-size scaling function}

Finally, in the vicinity of the critical point, we may write the following finite-size scaling form for the probability distribution of $\left(-\ln m_{1}^{s} m_{L}^{s}\right)$

$$
P_{L}\left(-\ln m_{1}^{s} m_{L}^{s}\right)=\frac{2}{\sqrt{\sigma L}} \mathcal{Q}\left(\mathcal{D}=\frac{-2 \ln m_{1}^{s} m_{L}^{s}}{\sqrt{\sigma L}} ; \gamma=\mu \sqrt{\sigma L}\right)
$$

where the scaling function reads

$$
\begin{aligned}
& \mathcal{Q}(\mathcal{D} ; \gamma)=\theta(\mathcal{D}>0) \theta(\mathcal{D}>-2 \gamma) \sum_{k=-\infty}^{+\infty} e^{-k^{2}(\mathcal{D}+\gamma)^{2}} \\
& {\left[k^{2}(\mathcal{D}+\gamma-|\gamma|)(2 k \mathcal{D}+(2 k-1) \gamma)^{2} e^{k \gamma(\mathcal{D}+\gamma)}+2 k(k-1)(2 k(\mathcal{D}+\gamma)-|\mu| \sigma L) e^{k|\gamma|(\mathcal{D}+\gamma)}\right]}
\end{aligned}
$$

By comparison with the results obtained before for the surface magnetization, we expect that the averaged correlation of the two surface magnetizations is governed by the exponent $\nu=2$ in the ordered phase and by the exponent $\tilde{\nu}=1$ in the disordered phase, for the reasons explained around Equation (90) : the scaling function (114) is defined in terms of the exponent $\nu=2$, but the presence of the theta function $\theta(\mathcal{D}>-2 \gamma)$ that forbids a finite interval in $\mathcal{D}$ near the origin in the disordered phase yields that the averaged value $\left[m_{1}^{s} m_{s}^{L}\right]_{\text {micro }}$ will be governed by the exponent $\tilde{\nu}=1$.

\section{Canonical ensemble}

For the canonical ensemble, it is more convenient to characterize the probability distribution of $D$ by its Laplace with respect to $L$ using the result (99)

$$
\begin{aligned}
& {\left[\hat{P}^{(\mu)}(D ; p)\right]_{\text {cano }}=\int_{0}^{+\infty} d L e^{-p L}\left[P_{L}^{(\mu)}(D)\right]_{\text {cano }}=\int d A \int d U \hat{P}^{(\mu)}(A, U ; p) \delta(D-(A-U))} \\
& =\theta(D>0) D \int_{1}^{+\infty} d v e^{\frac{\mu}{2} D(v-1)} \frac{q D \cosh q D v \cosh q D(v-1)-\sinh q D}{\sigma \sinh ^{3} q D v} \\
& +\theta(D>0) D \int_{1 / 2}^{1} d v e^{\frac{\mu}{2} D(v-1)} \frac{q D(2 v-1) \cosh q D v \cosh q D(1-v)+\sinh q D(1-2 v)}{\sigma \sinh ^{3} q D v}
\end{aligned}
$$

In particular at criticality $\mu=0$, the distribution of the variable $D$ takes the scaling form

$$
\left.\left[\mathcal{P}_{L}(D)\right)\right]_{\text {cano }}=\frac{1}{\sqrt{\sigma L}} \mathcal{P}_{\text {cano }}^{(0)}\left(\mathcal{D}=\frac{D}{\sqrt{\sigma L}}\right)
$$

where the scaling function is determined by the integral transform

$$
\begin{aligned}
\int_{0}^{+\infty} \frac{d \mathcal{D}}{\mathcal{D}^{2}} e^{-\frac{q^{2}}{\mathcal{D}^{2}}} \mathcal{P}_{\text {cano }}^{(0)}(\mathcal{D}) & =\int_{1}^{+\infty} d v \frac{q \cosh q v \cosh q(v-1)-\sinh q}{2 \sinh ^{3} q v} \\
& +\int_{1 / 2}^{1} d v \frac{q(2 v-1) \cosh q v \cosh q(1-v)-\sinh q(2 v-1)}{2 \sinh ^{3} q v}
\end{aligned}
$$


In particular, there is a logarithmic divergence in the limit $q \rightarrow 0$

$$
\int_{0}^{+\infty} \frac{d \mathcal{D}}{\mathcal{D}^{2}} e^{-\frac{q^{2}}{\mathcal{D}^{2}}} \mathcal{P}_{\text {cano }}^{(0)}(\mathcal{D}) \underset{q \rightarrow 0}{\simeq} \frac{1}{2} \ln \frac{1}{q}
$$

that yields the linear behavior near the origin

$$
\mathcal{P}_{\text {cano }}^{(0)}(\mathcal{D}) \underset{\mathcal{D} \rightarrow 0}{\propto} \mathcal{D}
$$

in contrast with the essential singularity found above for the microcanonical ensemble (108). As a consequence, the averaged correlation of the two surface magnetizations decays algebraically in $L$

$$
\left[m_{1}^{s} m_{L}^{s}\right]_{\text {cano }}^{\text {scaling }} \equiv \int_{0}^{+\infty} d \mathcal{D} P_{\text {cano }}(\mathcal{D}) e^{-\mathcal{D} \frac{\sqrt{\sigma L}}{2}} \underset{L \rightarrow \infty}{\propto} \frac{1}{L}
$$

in contrast with the stretched exponential behavior found for the microcanonical ensemble (109). This big difference between the two ensembles may be understood as follows : in the microcanonical ensemble where $U(L)=0$, the requirement to have a small $D$ is equivalent to the requirement to have a small amplitude $A=U_{\text {max }}-U_{\text {min }}$, which is a very strong constraint for a Brownian trajectory that leads to an essential singularity for the probability of small $D$. On the contrary, in the canonical ensemble, the requirement to have a small $D=A-U(L)$ is not equivalent to the requirement of a small amplitude $A$ : the freedom in $U(L)$ allows to have a large amplitude $A$ provided that $U(L)$ is of the same order : the samples having a small $D$ are thus those having $U_{\min } \sim 0$ ( probability $1 / \sqrt{L}$ ) and $U_{\max } \sim U(L)$ ( probability $1 / \sqrt{L}$ ) and thus the measure of these samples is of order $1 / L$, in agreement with the above result (121).

Finally, we note that the decay as $1 / L$ for the averaged correlation of the two surface magnetizations(121) in the canonical ensemble is similar to the decay found in [13] for the averaged spin-spin end-to-end correlation in the canonical ensemble. We will discuss the relation between these two observables in more details in the next Section.

\section{GAP AND END-TO-END SPIN-SPIN CORRELATION IN THE TWO ENSEMBLES}

In this Section, we consider the end-to-end spin-spin correlation and the gap in finite samples. In contrast with the surface magnetizations studied in previous Sections, these observables cannot be written in closed form in terms of all the random couplings. However, they have been studied in details in the canonical ensemble via the real-space renormalization approach [13], whose predictions are in good agreement with the numerical data 2, 13. The aim in this Section is to derive the corresponding results for the microcanonical ensemble.

\section{A. Gap and End-to-end spin-spin correlation in a given sample}

We refer the reader to the papers [7, 13] for a detailed description of the real-space renormalization approach. Here, we will only recall its results concerning the interpretation of the end-to-end spin-spin correlation $C(L)$ (4) and of the gap $\Delta(L)$ (5) for a given sample [13] : for a given realization of the random potential $U(x)$ for $0 \leq x \leq L$, the gap $\Delta(L)$ is asymptotically determined by the last renormalized ascending barrier $G$ via

$$
G=-\ln \Delta(L)
$$

and the correlation $C(L)$ is asymptotically determined by the variable $\Lambda=G-U(L)+U(0)$ via

$$
\Lambda=-\ln C(L)
$$

In particular, the RSRG predicts a very simple relation between the gap and the correlation in each given sample

$$
G-\Lambda=U(L)-U(0)
$$

This prediction has been confirmed by the numerical studies at the critical point, in the canonical sample where $(G-\Lambda) / \sqrt{L}$ was found to converge towards a Gaussian distribution [2, 13], as well as in the microcanonical sample where $(G-\Lambda) / \sqrt{L}$ was found to converge towards a delta distribution [2].

We refer the reader to [7, 13] for a full theoretical justification of these results. However, it is interesting to mention here that the RSRG prediction for $C(L)$ in the presence of a fixed boundary condition exactly corresponds to the 
saddle-point analysis of Section 【II for the surface magnetizations. Indeed, if the spin $\sigma_{L}=1$ is fixed, it cannot be decimated, and the last ascending barrier is constrained to contain this end-spin : the variable $G$ is thus determined by the minimum via $G=U(L)-U_{\min }$ and the variable $\Lambda$ reads $\Lambda=U_{\min }-U(0)$, in agreement with the saddle-point analysis of Section III for the surface magnetization $m_{1}^{s}$. When the boundary spins are both free, the gap and the correlation are not in general simple functions of the end-values $U(0)$ and $U(L)$ and of the extrema $U_{\min }$ and $U_{\max }$ of the random potential, but are determined by the properties of the last stage of the renormalization procedure as we now recall.

\section{B. Last stage of the renormalization procedure}

In the RSRG approach [], one needs at a given scale $\Gamma$ the measure $B_{\Gamma}^{ \pm}(F, l)$ of the ascending (resp. descending) Brownian paths that goes from $U(0)$ to $U(l)=F$ (resp. $U(l)=-F$ ) with $F \geq \Gamma$ and with no return of more than $\Gamma$, i.e. for two arbitrary points $0<x_{1}<x_{2}<l$, the potential has to satisfy $U\left(x_{1}\right)-U\left(x_{2}\right)<\Gamma\left(\right.$ resp. $\left.U\left(x_{2}\right)-U\left(x_{1}\right)<\Gamma\right)$ [35, 36]. In Laplace transform with respect to $l$ these measure read [7]

$$
B_{\Gamma}^{ \pm}(F ; p) \equiv \int_{0}^{+\infty} d l e^{-p l} B_{\Gamma}^{ \pm}(F, l) \quad=\theta(F-\Gamma) e^{ \pm \frac{\mu}{2} \Gamma} \frac{q}{\sinh q \Gamma} e^{-(F-\Gamma)\left(\mp \frac{\mu}{2}+q \operatorname{coth} q \Gamma\right)}
$$

where $q=\sqrt{p+\mu^{2} / 4}$ and $\sigma=1$ to simplify the notations in this Section. For the boundaries, one also needs the measure $E_{\Gamma}^{ \pm}(F, l)$ of the ascending (resp. descending) Brownian paths that goes from $U(0)$ to $U(l)=F$ where $F \geq 0$ is the maximum (resp. $U(l)=-F$ is the minimum) with no return of more than $\Gamma$. In Laplace transform with respect to $l$ these measure read [13, 35, 36]

$$
E_{\Gamma}^{ \pm}(F ; p) \quad=\theta(F) e^{-F\left(\mp \frac{\mu}{2}+q \operatorname{coth} q \Gamma\right)}
$$

The joint distribution $P_{L}(G, \Lambda, U)$ of the variables $G=-\ln \Delta(L), \Lambda=-\ln C(L)$ and of the end-point $U_{L}=U$ (with $U(0)=0$ ) is then determined by the last stage of the RSRG [13] as

$$
P_{L}(G, \Lambda, U)=\int d l_{1} d l_{2} d l_{3} d \Lambda_{R} d \Lambda_{L} E_{G}^{-}\left(\Lambda_{R}\right) B_{G}^{+}(G) E_{G}^{-}\left(\Lambda_{L}\right) \delta\left(L-\left(l_{1}+l_{2}+l_{3}\right)\right) \delta\left(\Lambda-\left(\Lambda_{L}+\Lambda_{R}\right)\right) \delta\left(U-(G-\Lambda) \ell_{12} 127\right)
$$

i.e. in Laplace with respect to $L$

$$
\hat{P}(G, \Lambda, U ; p)=\theta(G) \theta(\Lambda) \Lambda e^{-\Lambda\left(\frac{\mu}{2}+q \operatorname{coth} q G\right)} e^{\frac{\mu}{2} G} \frac{q}{\sinh q G} \delta(U-(G-\Lambda))
$$

\section{Canonical ensemble}

In the canonical ensemble, the joint distribution $P_{L}^{(\mu)}(G, \Lambda)$ has for Laplace transform

$$
\int_{0}^{+\infty} d L e^{-p L}\left[P_{L}^{(\mu)}(G, \Lambda)\right]_{\text {cano }}=\int d U \hat{P}(G, \Lambda, U ; p)=\theta(G) \theta(\Lambda) \frac{q \Lambda}{\sinh q G} e^{\frac{\mu}{2}(G-\Lambda)} e^{-\Lambda q \operatorname{coth} q G}
$$

which indeed coincides with the result (45) of [13]. We refer to [13] for a detailed analysis of this result, and only quote in the following some important results in order to compare them with the microcanonical case below.

\section{At criticality $\mu=0$}

At criticality, the law for the gap alone has for Laplace transform

$$
\int_{0}^{+\infty} d L e^{-p L}\left[P_{L}^{\mu=0}(G)\right]_{\text {cano }}=\theta(G) \frac{\sinh \sqrt{p} G}{\sqrt{p} \cosh ^{2} \sqrt{p} G}
$$

that corresponds after Laplace inversion to the series given in $\mathrm{Eq}(57)$ in 13]. In particular, the probability distribution of the rescaled variable $g=G / \sqrt{L}$ presents the essential singularity at the origin

$$
\mathcal{P}_{\text {cano }}^{\mu=0}(g) \underset{g \rightarrow 0}{\propto} \frac{1}{g^{3}} e^{-\frac{\pi^{2}}{4 g^{2}}}
$$


that determines the decay with $L$ of the averaged gap 13

$$
[\Delta(L)]_{\text {cano }}^{\text {scaling }} \underset{L \rightarrow \infty}{\propto} L^{1 / 6} e^{-\frac{3}{2}\left(\frac{\pi^{2}}{2} L\right)^{1 / 3}}
$$

On the other hand, the law for the correlation alone has for Laplace transform at criticality

$$
\int_{0}^{+\infty} d L e^{-p L}\left[P_{L}^{(\mu=0)}(\Lambda)\right]_{\text {cano }}=\theta(\Lambda) \sqrt{p} \Lambda \int_{0}^{+\infty} d G \frac{1}{\sinh \sqrt{p} G} e^{-\Lambda \sqrt{p} \operatorname{coth} \sqrt{p} G}=\theta(\Lambda) \Lambda K_{0}(\sqrt{p} \Lambda)
$$

which corresponds after Laplace inversion to the simple result for the rescaled variable $\lambda=\Lambda / \sqrt{L}$

$$
\mathcal{P}_{\text {cano }}^{\mu=0}(\lambda)=\theta(\lambda) \frac{\lambda}{2} e^{-\frac{\lambda^{2}}{4}}
$$

in excellent with numerical data [13]. As a consequence, the averaged correlation decays as a power of $L[13]$

$$
[C(L)]_{\text {cano }}^{\text {scaling }} \underset{L \rightarrow \infty}{\propto} \frac{1}{L}
$$

So in the canonical ensemble, at criticality, the gap and the spin-spin correlation have very different behaviors. In particular there is an essential singularity at the origin only for the gap and not for the correlation, and thus the decays for the averaged values are very different.

\section{Ordered phase $\mu=-2 \delta>0$}

In the ordered phase, the variable $\Lambda$ is simply the sum of two independent barriers $\left(U(0)-U_{\min }\right)$ and $\left(U_{\max }-U(L)\right)$ against the bias 13 ]

$$
P_{\text {cano }}^{(\mu>0)}(\Lambda) \simeq \mu^{2} \Lambda e^{-\mu \Lambda}
$$

whereas the variable $G=U(L)+\Lambda$ is asymptotically distributed with the Gaussian distribution of $U(L)$ alone 13$]$

$$
\begin{aligned}
& P_{\text {cano }}^{(\mu>0)}(G) \simeq \frac{1}{\sqrt{4 \pi L}} e^{-\frac{(G-\mu L)^{2}}{4 L}} \\
& \text { 3. Disordered phase } \mu=-2 \delta<0
\end{aligned}
$$

In the disordered phase, the probability distribution of the variable $G$ reads $[13]$

$$
P_{\text {cano }}^{(\mu<0)}(G) \simeq\left(L \mu^{2}\right)|\mu| e^{-|\mu| G} e^{-\left(L \mu^{2}\right) e^{-|\mu| G}}
$$

that can be interpreted [13] as the distribution of the maximal barrier among $N \sim L \mu^{2}$ independent variables drawn with the exponential distribution $|\mu| e^{-|\mu| G}$ of barriers against the drift. whereas the variable $\Lambda=-U(L)-G$ is asymptotically distributed with the Gaussian distribution of $U(L)$ alone [13]

$$
P_{\text {cano }}^{(\mu<0)}(\Lambda) \simeq \frac{1}{\sqrt{4 \pi L}} e^{-\frac{(\Lambda-|\mu| L)^{2}}{4 L}}
$$

\section{Microcanonical ensemble}

$$
\text { 1. At criticality } \mu=0
$$

At criticality $\mu=0$, we obtain that the the joint distribution $P_{L}^{(\mu=0)}(G, \Lambda)$ is determined by the Laplace transform

$$
\int_{0}^{+\infty} d L e^{-p L}\left[\frac{P_{L}^{(\mu=0)}(G, \Lambda)}{\sqrt{4 \pi L}}\right]_{\text {micro }}=\hat{P}(G, \Lambda, U=0 ; p)=\delta(\Lambda-G) \frac{\sqrt{p} G}{\sinh \sqrt{p} G} e^{-\sqrt{p} G \operatorname{coth} \sqrt{p} G}
$$


So here $G$ and $\Lambda$ are identical, and the rescaled variable

$$
g \equiv \frac{G}{\sqrt{L}}=\frac{\Lambda}{\sqrt{L}}
$$

is distributed with the distribution $\mathcal{P}_{\text {micro }}(g)$ determined by the integral transform

$$
\frac{1}{\sqrt{\pi}} \int_{0}^{+\infty} \frac{d g}{g} \mathcal{P}_{\text {micro }}(g) e^{-\frac{q}{g^{2}}}=\frac{\sqrt{q}}{\sinh \sqrt{q}} e^{-\sqrt{q} \operatorname{coth} \sqrt{q}}
$$

In particular, the following divergence near $q \rightarrow-\pi^{2}$

$$
\frac{1}{\sqrt{\pi}} \int_{0}^{+\infty} \frac{d g}{g} \mathcal{P}_{\text {micro }}(g) e^{-\frac{q}{g^{2}}} \underset{q \rightarrow \pi^{2}}{\propto} \frac{1}{q+\pi^{2}} e^{\frac{2 \pi^{2}}{q+\pi^{2}}}
$$

yields the following essential singularity for the probability distribution $\mathcal{P}_{\text {micro }}(g)$ at small argument

$$
\mathcal{P}_{\text {micro }}(g) \underset{g \rightarrow 0}{\propto} \frac{1}{g^{3 / 2}} e^{-\frac{\pi^{2}}{g^{2}}+\frac{2 \pi \sqrt{2}}{g}}
$$

This behavior at small $g$ of the scaling function characterizes the rare events having a gap $\Delta_{L}=e^{-g \sqrt{L}}$ (or equivalently a correlation $C_{L}=e^{-g \sqrt{L}}$ ) of order one, which are expected to dominate the mean values of the gap and of the correlation. The dependence in $L$ of these mean values may thus be estimated by a saddle point method which yields

$$
\begin{aligned}
{\left[\Delta_{L}\right]_{\text {micro }}^{\text {scaling }}=\left[C_{L}\right]_{\text {micro }}^{\text {scaling }} } & \simeq \int_{0}^{+\infty} d g \mathcal{P}_{\text {micro }}(g) e^{-g \sqrt{L}} \\
& \stackrel{\propto}{\propto} L^{-1 / 6} e^{-\frac{3}{2}\left(2 \pi^{2} L\right)^{1 / 3}+2\left(2 \pi^{2} L\right)^{1 / 6}}
\end{aligned}
$$

So here we obtain that there is a sub-leading power $L^{1 / 6}$ in the exponential with respect to the leading decay as $E^{-c L^{1 / 3}}$, in contrast with the canonical ensemble (132).

$$
\text { 2. } \text { Ordered phase } \mu=-2 \delta>0
$$

In the ordered phase, the variable $\Lambda$ is again the sum of two independent barriers $\left(U(0)-U_{\min }\right)$ and $\left(U_{\max }-U(L)\right)$ against the bias, as in the canonical ensemble (136). This distribution of $\Lambda$ now completely determines the probability distribution of the variable $G=\mu L+\Lambda$, in contrast with the Gaussian distribution of the canonical ensemble (136). In particular, the inequality $G>\mu L$ indicates that the average of the gap $\Delta(L)=e^{-G}$ will be governed by the exponent $\tilde{\nu}=1$ instead of the exponent $\nu=2$, for the reasons given around Equation (90) concerning the case of the averaged surface magnetization.

\section{Disordered phase $\mu=-2 \delta<0$}

In the disordered phase, the distribution of the variable $G$ will be asymptotically the same as in the canonical ensemble (138), since the interpretation [13] as the the maximal barrier among $N$ independent barriers against the drift still holds in the microcanonical ensemble. However, the distribution of the variable $\Lambda$ is now completely determined by the relation $\Lambda=|\mu| L+G$, in contrast with the Gaussian distribution (138) of the canonical case. In particular, the inequality $\Lambda>|\mu| L$ indicates that the average of the spin-spin correlation $C(L)=e^{-\Lambda}$ will be governed by the exponent $\tilde{\nu}=1$ instead of the exponent $\nu=2$, for the reasons given around Equation (90) concerning the case of the averaged surface magnetization.

\section{E. Comparison between $C(L)$ and $m_{1}^{s} m_{L}^{s}$ at the critical point}

In the ordered phase, where the two end spins have a spontaneous magnetization, it is clear that the limit $C(\infty)$ corresponds to the product of two independent end-point magnetizations of an infinite system 13. Moreover, it was found numerically [2] that the observables $C(L)$ and $\left(m_{1}^{s} m_{L}^{s}\right)$ were actually still closely related at the critical point 
: the limit distributions were found to be very close in the microcanonical ensemble, but different at large argument in the canonical ensemble 2]. On the other hand, the difference at small argument is much smaller in the canonical ensemble, and the average values $[C(L)]_{\text {cano }}$ and $\left[m_{1}^{s} m_{L}^{s}\right]_{\text {cano }}$ were found to be almost indistinguishable numerically in the canonical ensemble [2]. It is thus interesting to discuss these questions here.

From the RSRG approach, it is clear that if the positions $x_{\min }$ and $x_{\max }$ of the minimum $U_{\min }$ and $U_{\max }$ satisfy the order $0 \leq x_{\min }<x_{\max } \leq L$, then the largest ascending barrier is simply given by the amplitude $G=U_{\max }-U_{\min }$. Then the variable $\Lambda$ for the spin-spin correlation is given by $\Lambda=\left(U_{\max }-U(L)\right)+\left(U(0)-U_{\text {min }}\right)$ and thus exactly coincides with the variable $D=A(L)-U(L)$ (97) determining the correlation of the two surface magnetizations. In particular, in the ordered phase, all samples satisfy $0<x_{\min }<x_{\max }<L$ in the asymptotic limit $L \rightarrow \infty$, and the variables $\Lambda=D$ are the sum of two independent barriers against the bias. At the critical point, half of the samples satisfy the constraint $x_{\min }<x_{\max }$, and thus we obtain that for one half of the samples at criticality, both in the canonical and in the microcanonical ensemble we have the identity $\Lambda=D$ that yields the identity of the two rescaled variables $(\ln C(L)) / \sqrt{L}=\left(\ln m_{1}^{s} m_{L}^{s}\right) / \sqrt{L}$. On the other hand, for the other half of the samples which have $x_{\max }<x_{\min }$, the largest ascending barrier is smaller than the amplitude $G<U_{\max }-U_{\min }$ and thus $\Lambda<D$ are two different variables. In conclusion, the identity $\Lambda=D$ for half of the samples could explain the similarities found numerically in 2] for their probability distributions.

Concerning the mean values, the interpretation given after Equation (121) for the decay as $1 / L$ of the averaged correlation of the surface magnetizations in the canonical ensemble yields that the relevant rare events precisely satisfy the order $0 \leq x_{\min }<x_{\max } \leq L$. As a consequence, we expect that the leading term in $1 / L$ of the averaged spin-spin correlation exactly coincides with the leading term in $1 / L$ of the averaged correlation of the surface magnetizations in the canonical ensemble, in agreement with the numerical finding that both quantities were almost indistinguishable [2].

\section{CONCLUSIONS}

The example of the RTFIC considered in this paper shows that the question of the ensemble dependence for the critical properties of disordered systems is rich and instructive. Indeed, even if the two ensembles are expected to be equivalent in the thermodynamic limit, their finite-size properties may nevertheless be quite different.

At criticality, even if the appropriate rescaled variables for all observables considered here are the same in both ensembles, the probability distributions may be quite different in the two ensembles. In particular, some asymptotic behavior may be given by two different power-laws in the two ensembles (as in the case of the surface magnetization), or even by a power law in one ensemble and by an essential singularity in the other ensemble (as in the case of the end-to-end correlations). As a consequence, the decay with $L$ of averaged observables can be drastically different in the two ensembles : for instance, the end-to-end correlations decay as a power law in the canonical ensemble, but as a stretched exponential in the microcanonical ensemble.

Off criticality, the probability distributions of rescaled variables involve the exponent $\nu=2$ in both ensembles, but averaged observables are again governed by rare samples, whose measures can be very sensitive to the microcanonical constraint. As a consequence, the critical properties of a given averaged observable may be governed by two different exponents in the two ensembles. For instance, we have shown via an exact path-integral method that the averaged surface magnetization in the disordered phase was governed by the exponent $\nu=2$ in the canonical ensemble and by the exponent $\tilde{\nu}=1$ in the microcanonical ensemble, in agreement with the interpretation of [1], and in contrast with the interpretation of [2]. We have moreover concluded from the domains of definition of probability distributions of other observables in the scaling regime, that similarly, (a) in the disordered phase, the averaged correlation of the two surface magnetizations and the end-to-end spin-spin correlation are governed by the exponent $\nu=2$ in the canonical ensemble and by the exponent $\tilde{\nu}=1$ in the microcanonical ensemble (b) in the ordered phase, the averaged gap is governed by the exponent $\nu=2$ in the canonical ensemble and by the exponent $\tilde{\nu}=1$ in the microcanonical ensemble.

As a final remark, let us mention the very recent preprint [37], where the RSRG method is generalized to study the end-to-end energy-energy correlation $C_{E}(L)$ (in the canonical ensemble), and where averaged observables are again governed by rare events.

\section{Acknowledgments}

It is a pleasure to thank T. Garel and J. Houdayer for useful discussions. 


\section{APPENDIX A: JOINT DISTRIBUTION OF THE AMPLITUDE $A$ AND THE END-POINT $U$}

The probability $G_{\left[U_{a}, U_{b}\right]}\left(U, U_{0} ; l\right)$ for a random walk with bias $F_{0}=\mu \sigma$ to go from $U_{0}=0$ to $U$ during $L$ in the presence of two absorbing boundaries at $U=U_{a}<0$ and $U=U_{b}>0$ has for Laplace transform [35, 36]

$$
\begin{array}{ll}
\hat{G}_{\left[U_{a}, U_{b}\right]}^{\mu}\left(U, p \mid U_{0}\right)=e^{\frac{\mu}{2}\left(U-U_{0}\right)} \frac{\sinh q\left(U-U_{a}\right) \sinh q\left(U_{b}-U_{0}\right)}{\sigma q \sinh q\left(U_{b}-U_{a}\right)} \quad \text { if } \quad U_{a} \leq U \leq U_{0} \\
\hat{G}_{\left[U_{a}, U_{b}\right]}^{\mu}\left(U, p \mid U_{0}\right)=e^{\frac{\mu}{2}\left(U-U_{0}\right)} \frac{\sinh q\left(U_{b}-U\right) \sinh q\left(U_{0}-U_{a}\right)}{\sigma q \sinh q\left(U_{b}-U_{a}\right)} \quad \text { if } \quad U_{0} \leq U \leq U_{b}
\end{array}
$$

where

$$
q \equiv \sqrt{\frac{p}{\sigma}+\frac{\mu^{2}}{4}}
$$

The joint probability of the end-point $U$, of the minimum value $U_{a}$ and of the maximal value $U_{b}$ when starting at $U_{0}=0$ has thus for Laplace transform

$$
\begin{aligned}
& \hat{P}^{(\mu)}\left(U, U_{a}, U_{b} ; p\right)=-\partial_{U_{a}} \partial_{U_{b}} \hat{G}_{\left[U_{a}, U_{b}\right]}^{\left(F_{0}\right)}(U, p \mid 0) \\
& =e^{\frac{\mu}{2} U} \frac{q}{\sigma} \frac{\sinh q U \sinh q\left(U_{b}+U_{a}\right)-2 \cosh q U \sinh q U_{a} \sinh q U_{b}-}{\sinh ^{3} q\left(U_{b}-U_{a}\right)}
\end{aligned}
$$

Finally, the joint probability of the amplitude $A_{L}=U_{\max }-U_{\min }=U_{b}-U_{a}$ and the end-point $U$ when starting at $U_{0}=0$ has for Laplace transform

$$
\begin{aligned}
\hat{P}^{(\mu)}(A, U ; q) & =\int_{-\infty}^{+\infty} d U_{a} \int_{-\infty}^{+\infty} d U_{b} \theta\left(U_{a}<0<U_{b}\right) \theta\left(U_{a}<U<U_{b}\right) \hat{P}^{(\mu)}\left(U, U_{a}, U_{b}\right) \delta\left(A-\left(U_{b}-U_{a}\right)\right) \\
& =\theta(A>|U|) \int_{\max (-A, U-A)}^{\min (0, U)} d U_{a} \hat{P}^{(\mu)}\left(U, U_{a}, U_{a}+A\right)
\end{aligned}
$$

This finally leads to the result (99) given in the text.

\section{APPENDIX B: USEFUL PROPERTIES OF BESSEL FUNCTIONS}

The series expansion of Bessel function at small argument reads

$$
K_{i q}(z)=\frac{\pi}{2 i \sinh \pi q} \sum_{k=0}^{+\infty} \frac{\left(\frac{z}{2}\right)^{2 k}}{k !}\left[\frac{\left(\frac{z}{2}\right)^{-i q}}{\Gamma(-i q+k+1)}-\frac{\left(\frac{z}{2}\right)^{i q}}{\Gamma(i q+k+1)}\right]
$$

whereas the asymptotic behavior at large argument is given by

$$
K_{\nu}(z) \underset{z \rightarrow \infty}{\simeq} \sqrt{\frac{\pi}{2 z}} e^{-z}\left[\sum_{k=0}^{n} \frac{1}{k !(2 z)^{k}} \frac{\Gamma\left(\nu+k+\frac{1}{2}\right)}{\Gamma\left(\nu-k+\frac{1}{2}\right)}+. .\right]
$$

A useful integral is

$$
\int_{0}^{+\infty} d p p^{\frac{\mu}{2}-1} e^{-p} K_{i q}\left(2 \sqrt{\frac{p}{\sigma}}\right)=\frac{\sqrt{\sigma}}{2} e^{\frac{1}{2 \sigma}} \Gamma\left(\frac{\mu+1+i q}{2}\right) \Gamma\left(\frac{\mu+1-i q}{2}\right) W_{-\frac{\mu}{2}, i \frac{q}{2}}\left(\frac{1}{\sigma}\right)
$$

where $W$ is the Whittaker function. For the special case $\mu=0$, it simplifies into

$$
W_{0, r}(z)=\sqrt{\frac{z}{\pi}} K_{r}\left(\frac{z}{2}\right)
$$

[1] F. Igloi and H. Rieger. Phys. Rev. B 57 (1998) 11404. 
[2] A. Dhar and A.P. Young, cond-mat/0305664

[3] F. Pazmandi, R. Scalettar and G. Zimanyi, Phys. Rev. Lett. 79 (1997) 5130.

[4] K. Bernardet, F. Pazmandi and G. Batrouni, Phys. Rev. Lett. 84 (2000) 4477.

[5] S. Wiseman and E. Domany, Phys. Rev. Lett. 81 (1998) 22; Phys Rev E 58 (1998) 2938.

[6] A. Aharony, A.B. Harris and S. Wiseman, Phys. Rev. Lett. 81 (1998) 252.

[7] D.S. Fisher, Phys. Rev. B 516411 (1995).

[8] B. M. McCoy and T. T. Wu, Phys. Rev. 176, 631 (1968) and Phys. Rev. 188, 982 (1969) ; B. M. McCoy, Phys. Rev. 188, 1014 (1969) and Phys. Rev. B 2, 2795 (1970).

[9] R. Shankar and G. Murthy, Phys. Rev. B 36536 (1987).

[10] A.B. Harris, J. Phys. C 7 (1974) 1671.

[11] Y. Imry and S. Ma, Phys. Rev. Lett. 35 (1975) 1399.

[12] J.T. Chayes, L. Chayes, D.S. Fisher and T. Spencer, Phys. Rev. Lett. 57 (1986) 2999.

[13] D.S. Fisher and A. P. Young, Phys. Rev. B 58, 9131-9141 (1998).

[14] H. Kesten, M. Koslov and F. Spitzer, Composio. Math. 30 (1975) 145.

[15] F. Solomon, Ann. Prob. 3 (1975) 1.

[16] Y. Sinai, Theor. Prob. Appl. 27 (1982) 256.

[17] B. Derrida and Y. Pomeau, Phys. Rev. Lett 48 (1982) 627; B. Derrida, J. Stat. Phys. 31 (1983) 433.

[18] V. Afanasev, Theor. Prob. Appl. 35 (1990) 205.

[19] B. Derrida and H.J. Hilhorst, J. Phys. A 16 (1983) 7183.

[20] C. Calan, J.M. Luck, T. Nieuwenhuizen and D. Petritis, J. Phys. A 18 (1985) 501.

[21] H. Kesten, Acta Math. 131 (1973) 207.

[22] J. P. Bouchaud, A. Comtet, A. Georges and P. Le Doussal, Ann. Phys. 201 (1990) 285.

[23] D.S. Fisher, P. Le Doussal and C. Monthus, Phys. Rev. Lett. 803539 (1998); P. Le Doussal, C. Monthus, D.S. Fisher, Phys. Rev. E 594795 (1999).

[24] F. Igloi, R. Juhasz, and H. Rieger Phys. Rev. B 59, 11308 (1999); F. Igloi, R. Juhasz and P. Lajko, Phys. Rev. Lett. 86 1343 (2001); F. Igloi, Phys. Rev. B 6564416 (2002).

[25] C. Monthus and A. Comtet, J. Phys. I (France) 4 (1994) 635.

[26] S. F. Burlatsky, G. S. Oshanin, A. V. Mogutov, and M. Moreau Phys. Rev. A 45 (1992) R6955.

[27] G. Oshanin, S.F. Burlatsky, M. Moreau and B. Gaveau, J. Stat. Phys. 73 (1993).

[28] C. Monthus and P. Le Doussal, Phys. Rev. E 65 (2002) 66129.

[29] M.V. Feigelman and V. Vinokur, J. Phys. (France) 491731 (1988)

[30] S. Redner, Am. J. Phys. 58 (1990) 267.

[31] A. Comtet, C. Monthus and M. Yor, J. Appl. Prob. 35 (1998) 255.

[32] M. Yor, "Exponential functionals of Brownian motion and related processes", Springer (2001).

[33] L. Balents and M. P. A. Fisher, Phys. Rev. B 56, 12970-12991 (1997).

[34] O. Motrunich, K. Damle and D.A. Huse, Phys. Rev. B 63134424 (2001).

[35] P. Le Doussal and C. Monthus, Physica A 317 (2003) 140-198.

[36] C. Monthus and P. Le Doussal, cond-mat/0206035

[37] G. Refael and D. S. Fisher, cond-mat/0308176 